\title{
BOOT LIMIT: UMA APLICAÇÃO EM RADIOQUÍMICA
}

SILVA, Cleomacio Miguel da ${ }^{1}$ VIEIRA, José Wilson ${ }^{1}$

COSTA JÚNIOR, Carlos Eduardo de Oliveira ${ }^{2}$

RESUMO: O objetivo do presente trabalho foi utilizar o método bootstrap para construir intervalos de confiança para limite de detecção em estudos radioquímicos. Para tanto, foi desenvolvido um programa computacional na linguagem $\mathrm{C}++$ que foi denominado de boot limit. Para testar o boot limit, determinou-se a concentração de ${ }^{210} \mathrm{~Pb}$ em condições de background (branco) usando o método da resina trocadora de íons em amostras de água deionizada. Neste caso, o limite de detecção variou de 26,7 a $140,7 \mathrm{mBq} \cdot \mathrm{L}^{-1}$, com média de $70,34 \mathrm{mB} \cdot \mathrm{L}^{-1}$. Os resultados mostraram que o método bootstrap é uma ferramenta estatística bastante eficiente para construir intervalos de confiança na determinação do limite de detecção em radioquímica.

Palavras-chave: Estatística Inferencial. Modelagem Matemática. Monte Carlo.

\section{BOOT LIMIT: AN APPLICATION IN RADIOQUÍMICA}

SUMMARY: The objective of the present study was to use the bootstrap method to construct confidence intervals for limit detection in radiochemical studies. For that, a computer program was developed in the $\mathrm{C}++$ language that was called boot limit. To test the boot limit, the concentration of ${ }^{210} \mathrm{~Pb}$ under background conditions (white) was determined using the ion exchange resin method in deionized water samples. In this case, the limit of detection ranged from 26.7 to $140.7 \mathrm{mBq} . \mathrm{L}^{-1}$, with a mean of $70.34 \mathrm{mB} . \mathrm{L}^{-1}$. The results showed that the bootstrap method is a very efficient statistical tool to construct confidence intervals in determining the limit of detection in radiochemistry.

Keywords: Inferential Statistics. Mathematical Modeling. Monte Carlo.

\section{INTRODUÇÃO}

Toda técnica analítica possui uma quantidade ou concentração mínima do analito que pode ser detectada. Isso remete a um conceito oriundo das medições quantitativas da química analítica, o limite de detecção. Geralmente, a literatura define o limite detecção como a menor quantidade ou concentração de um analito que pode ser estabelecida como diferente, admitindo-se um intervalo de confiança, em relação a um branco (um material similar a amostra em termos de composição, exceto pelo fato do analito estar ausente). Uma definição de limite de detecção amplamente encontrada na literatura é a concentração do analito que produz um sinal que excede em três vezes o desvio padrão do sinal obtido quando a amostra de background (branco) é analisada. Assim, o limite de detecção é uma função do número de vezes que submeteram a amostra de branco à análise, já que o cálculo do desvio padrão depende dessa amostra (WEHRY, 1997). Devido à natureza estatística que há no cálculo do limite de detecção, há ocasiões em que o analito está ausente na amostra e, no entanto, é considerado como presente (situação denominada "falso positivo"). Em outras, o analito está presente, mas é considerado como ausente (situação

\footnotetext{
${ }^{1}$ Escola Politécnica da Universidade de Pernambuco

${ }^{2}$ Instituto Federal de Pernambuco
} 
denominada "falso negativo"). Devido a isso, amostras com concentrações do analito iguais ou próximas à do limite de deteç̧ão são consideradas como tendo determinação imprecisa. Essa incerteza faz com que tomemos a decisão de não quantificarmos o analito a menos que sua concentração esteja bem acima do limite de detecção. Desse modo, devido ao cálculo estatístico necessário para a obtenção do limite de detecção, ele não deve ser entendido como uma concentração única, mas sim uma faixa de concentrações que gera incerteza na determinação analítica (WEHRY, 1997).

O limite de detecção é uma grandeza fundamental em radioquímica, pois, é parte integrante do processo de validação do método analítico. Assim, o limite de detecção deve garantir, por meio de estudos experimentais, que o método atenda às exigências das aplicações analíticas, assegurando a confiabilidade dos resultados. Para a garantia da qualidade analítica dos resultados, todos os equipamentos utilizados na validação devem estar devidamente calibrados e os analistas devem ser qualificados e adequadamente treinados. A utilização do limite de detecção para decidir se um analito está presente numa amostra é conceitualmente simples, mas o tema ainda gera confusão e discordância entre diversos autores. $\mathrm{O}$ limite de detecção tem sido utilizado em radioquímica, pelo menos, desde 1962. As primeiras publicações sobre o tema foram realizadas por Altshuler e Pasternack (1963), Nicholson (1963 e 1966) e Currie (1968). Por outro lado, trabalhos mais recentes realizados pela IUPAC (1995), ISO (2010), ANVISA (2003) e INMETRO (2003) fizeram estimativas de limite de detecção abordando diferentes conceitos estatísticos.

A literatura apresenta diversos modelos estatísticos que são utilizados para calcular o limite de detecção. Nos trabalhos realizados por Altshuler e Pasternack (1963), Nicholson (1963 e 1966), Currie (1968), IUPAC (1995), ANVISA (2003) e INMETRO (2003) utilizaram-se a estatística tradicional baseado na distribuição de Poisson. Por outro lado, a ISO (2010) utiliza a distribuição de Poisson com ajustes realizados pela estatística bayesiana. Porém, em tais situações, considera-se que a distribuição de Poisson tende para a distribuição normal para valores suficientemente grandes. Entretanto, muitas avaliações na teoria da amostragem são de uso limitado quando o conjunto dos dados é pequeno. Por exemplo, a estimativa do desvio padrão para $n$ valores normalmente distribuídos, $\sqrt{\frac{1}{(n-1)} \sum\left(\mathrm{x}_{\mathrm{i}}-\overline{\mathrm{x}}\right)^{2}}$ é muito sensível às flutuações quando $\mathrm{n}$ é pequeno do que quando ele é grande. A aplicação do método bootstrap para calcular a média aritmética de dados de pequenas amostras não difere da utilização dos procedimentos usuais, pois, os estimadores bootstrap constituem-se uma combinação linear dos valores experimentais (HELENE; VANIN, 2002). Por outro lado, o Teorema do Limite Central garante que, quando $\mathrm{n}$ for muito grande, os estimadores da média e dos desvios são normalmente distribuídos e convergem para os seus valores verdadeiros (EFRON, 1982). Sendo assim, dentro desse contexto, o objetivo deste trabalho foi aplicar o método estatístico bootstrap para construir intervalos de confiança na determinação do limite de detecção nas análises radioquímicas.

\section{MATERIAL E MÉTODO}

\section{Determinação do ${ }^{210} \mathrm{~Pb}$}

Utilizou-se o método da troca iônica apresentado por Godoy et al. (1998), para determinar as concentrações de ${ }^{210} \mathrm{~Pb}$ nas amostras de água deionizada. Primeiramente, utilizou-se $1 \mathrm{~L}$ de água que foi colocada para evaporar à temperatura de $60{ }^{\circ} \mathrm{C}$, até a redução de $200 \mathrm{~mL}$. Após este procedimento, as amostras foram colocadas para lixiviar com $100 \mathrm{~mL}$ de $\mathrm{HBr} 0,5 \mathrm{M}$ e $1,0 \mathrm{~g}$ de cloridrato de hidroxilamina, por um período de 12 horas. A solução foi filtrada e adicionou-se $1 \mathrm{~mL}$ de carreador de $\mathrm{Pb}^{++}(20 \mathrm{mg} / \mathrm{L}$ de $\left.\mathrm{Pb}\left(\mathrm{NO}_{3}\right)_{2}\right)$. Depois, a solução foi colocada para percolar numa coluna contendo resina trocadora de íons do 
tipo BIO-RAD DOWEX 1-X8 50-100 mesh chloride form. Durante esta etapa, o ${ }^{210} \mathrm{~Pb}$ fica retido na resina, sendo depois, eluido com $50 \mathrm{~mL}$ de $\mathrm{HNO}_{3} 1 \mathrm{M}$. A solução obtida foi aquecida até a secagem total. Adicionou-se $50 \mathrm{~mL}$ de água deionizada e corrigiu-se o $\mathrm{pH}$ (que deve estar entre 4,5 a 5,0) com acetato de amônio a $40 \%$. A solução foi aquecida até a ebulição, e depois adicionou $2 \mathrm{~mL}$ de $\mathrm{Na}_{2} \mathrm{CrO}_{4}$ a $25 \%$. A solução foi filtrada em papel quantitativo, cobrindo-se o precipitado com papel de filtro milipore, que em seguida foi levado para o detector para determinar a contagem beta. A concentração de ${ }^{210} \mathrm{~Pb}\left(\mathrm{C}_{\mathrm{Pb}-210}\right) \mathrm{em}$ Bq. $\mathrm{L}^{-1}$ foi determinada utilizando a Equação (GODOY et al., 1998).

$$
\mathrm{C}_{\mathrm{Pb}-210}=\frac{\mathrm{A}_{\mathrm{L}}}{\left(1-\mathrm{e}^{-\lambda_{\mathrm{Bi}} \mathrm{t}}\right) \eta \mathrm{yW}}
$$

Onde:

$\mathrm{A}_{\mathrm{L}}=$ contagem líquida obtida no detector $(\mathrm{cpm})$;

$\mathrm{t}=$ tempo do crescimento do $\mathrm{Bi}$ (bismuto);

$\lambda_{\mathrm{Bi}}=$ constante de decaimento do $\mathrm{Bi}\left(\mathrm{dias}^{-1}\right)$;

$\eta=$ eficiência de detecção do $\mathrm{Bi}(\mathrm{cpm} / \mathrm{Bq})$;

$\mathrm{y}=$ rendimento químico $(\%)$;

$\mathrm{w}=$ quantidade de água deionizada utilizada na análise $(\mathrm{L})$

\section{Preparação das amostras padrões}

$\mathrm{Na}$ avaliação do método da troca iônica, realizaram-se procedimentos de intercomparação no Laboratório de Monitoração Ambiental do Departamento de Energia Nuclear da Universidade Federal de Pernambuco. Para tanto, utilizaram-se padrões líquidos certificados de ${ }^{210} \mathrm{~Pb}$ fornecidos pelo Instituto de Radioproteção e Dosimetria (IRD). Os padrões líquidos foram diluídos em ácido nítrico 1 N, conformes recomendações do IRD. Inicialmente, prepararam-se amostras de água deionizada, semelhantemente aos procedimentos experimentais adotados na análise. Em cada amostra foram adicionados padrões de ${ }^{210} \mathrm{~Pb}$, sendo então, submetidas aos procedimentos experimentais apresentados no item anterior. Nesse caso, devido ao elevado grau de exigência radioanalítica, foram seguidos os procedimentos de validação de métodos analíticos e bioanalíticos, como estabelecidos pela Agência Nacional de Vigilância Sanitária (ANVISA) do Ministério da Saúde do Brasil (2003).

\section{Análise estatísticas dos dados}

$\mathrm{Na}$ análise estatística dos dados foi utilizado o método bootstrap. O método bootstrap foi introduzido por Efron (1982) e, desde então, tem sido profundamente estudado, não apenas em estudos teóricos, como também em várias aplicações. Todavia, a necessidade de manipulação de um número geralmente grande de amostras, a sua operacionalidade somente tornou-se viável com o advento e popularização dos microcomputadores. O método consiste num procedimento estatístico computacionalmente intensivo que permite a avaliação de diversas estatísticas, com base nos dados obtidos da amostra. Sendo assim, ele tem como base a ideia de que o pesquisador pode tratar a sua amostra como se fosse a população que deu origem aos dados e utilizar amostragem com reposição de sua amostra experimental para gerar pseudo-amostras, e a partir destas, estimar características de interesse de certas estatísticas (EFRON, 1982). Nesse caso, a inferência estatística bootstrap tem a finalidade de produzir afirmações sobre uma dada característica da população de interesse, a partir de informações colhidas da amostra. 
Vários esquemas diferentes de bootstrap têm sido propostos e muitos deles apresentam bom desempenho em uma ampla variedade de situações. Esse método pode ser implementado tanto na estatística não-paramétrica quanto na paramétrica, dependendo apenas do conhecimento do problema. No caso não-paramétrico, reamostra-se os dados com reposição, de acordo com uma distribuição empírica estimada, tendo em vista que, no geral, não se conhece a distribuição subjacente aos dados. No caso paramétrico, quando se tem informação suficiente sobre a forma da distribuição dos dados, a amostra bootstrap é formada realizando-se a amostragem diretamente nessa distribuição com os parâmetros desconhecidos substituídos por estimativas paramétricas.

O processo de reamostragem consiste em gerar amostras a partir da amostra original, cujos dados aleatoriamente retirados (com reposição) são utilizados na formação de cada amostra bootstrap. Dessa forma, todo resultado depende diretamente da amostra original. A distribuição da estatística de interesse aplicada aos valores desse tipo de amostragem, condicional aos dados observados, é definida como a distribuição bootstrap dessa estatística (BICKEL; FREEDMAN, 1981). Operacionalmente, o procedimento bootstrap consiste na reamostragem de mesmo tamanho e com reposição dos dados da amostra original, e cálculo da estatística de interesse para cada reamostra, denominada de pseudo-valores (BICKEL; FREEDMAN, 1981). Efron e Tibshirani (1993) apresentaram as ideias básicas subjacentes ao método de bootstrap, no âmbito da inferência clássica da estatística, como se segue. Com $\mathrm{x}=\left(\mathrm{x}_{1}, \mathrm{x}_{2}, \cdots, \mathrm{x}_{\mathrm{n}}\right)$ amostra aleatória obtida a partir de uma população com função de distribuição desconhecida, F, seja, $\hat{\Theta}\left(x_{1}, x_{2}, \cdots, x_{n}\right)$, um estimador do parâmetro $\Theta(F)$ que, como se indica, depende naturalmente de F. Seja $\hat{\mathrm{F}}$ a função de distribuição empírica associada à amostra obtida, tal que a cada valor observado $\mathrm{x}_{\mathrm{i}}$, onde $(\mathrm{i}=1,2, \cdots, \mathrm{n})$, atribui massa probabilística $\frac{1}{\mathrm{n}}$. Então, o valor de $\hat{\mathrm{F}}$ é calculado pela Equação 2.

$$
\hat{\mathrm{F}}_{(\mathrm{n})}(\mathrm{x})=\frac{\left[\sum_{\mathrm{i}=1}^{\mathrm{n}} \mathrm{I}\left(\mathrm{x}_{\mathrm{i}} \leq \mathrm{x}\right)\right]}{\mathrm{n}}
$$

Onde:

$\hat{\mathrm{F}}_{(\mathrm{n})}(\mathrm{x})$ - o estimador não-paramétrico de máxima verossimilhança de $\mathrm{F}$;

$\mathrm{I}\left(\mathrm{x}_{\mathrm{i}} \leq \mathrm{x}\right)$ - função indicadora.

Uma amostra bootstrap é uma amostra $\mathrm{x}^{*}=\left(\mathrm{x}_{1}^{*}, \mathrm{x}_{2}^{*}, \cdots, \mathrm{x}_{\mathrm{n}}^{*}\right)$ obtida de forma aleatória e com reposição a partir da amostra original $\mathrm{x}=\left(\mathrm{x}_{1}, \mathrm{x}_{2}, \cdots, \mathrm{x}_{\mathrm{n}}\right)$, também designada população bootstrap. A notação com asterisco indica que ${ }^{*}$ não é um novo conjunto de dados reais $\mathrm{x}$, mas sim uma versão randomizada, ou reamostrada de $\mathrm{x}$. A amostra bootstrap consiste dos correspondentes membros de $\mathrm{x}$, onde: $\mathrm{x}_{1}^{*}=\mathrm{x}_{\mathrm{i} 1}, \mathrm{x}_{2}^{*}=\mathrm{x}_{\mathrm{i} 2}, \cdots, \mathrm{x}_{\mathrm{n}}^{*}=\mathrm{x}_{\mathrm{in}}$. $\mathrm{O}$ conjunto $\left(\mathrm{x}_{\mathrm{i} 1}{ }^{*}, \mathrm{x}_{\mathrm{i} 2}{ }^{*}, \ldots, \mathrm{x}_{\mathrm{in}}^{*}\right)$ representa a i-ésima amostra de tamanho $\mathrm{n}$ com reposição dos dados originais do conjunto $\mathrm{x}=\left(\mathrm{x}_{1}, \mathrm{x}_{2}, \cdots, \mathrm{x}_{\mathrm{n}}\right)$. No método bootstrap, a média amostral calculada é denominada por $\overline{\mathrm{x}}_{\mathrm{i}}$. A cada procedimento de reamostragem do conjunto original $\mathrm{x}=\left(\mathrm{x}_{1}, \mathrm{x}_{2}, \cdots, \mathrm{x}_{\mathrm{n}}\right)$, correspondem estimadores, dados por $\overline{\mathrm{x}}_{1}, \overline{\mathrm{x}}_{2}, \ldots, \overline{\mathrm{x}}_{\mathrm{n}}$. Neste caso, o estimador bootstrap da média da população é a média aritmética, $\overline{\mathrm{x}}_{\mathrm{B}}$, dos n estimadores $\overline{\mathrm{x}}_{\mathrm{i}}$. Sendo este, um método de reamostragem com reposição, pode-se ter, por exemplo: $\mathrm{x}_{1}^{*}=\mathrm{x}_{7}, \mathrm{x}_{2}^{*}=\mathrm{x}_{10}, \mathrm{x}_{3}^{*}=\mathrm{x}_{2}, \cdots, \mathrm{x}_{\mathrm{B}}^{*}=\mathrm{x}_{3}$. Portanto, o conjunto de dados reamostrados é constituído de elementos do conjunto dos dados originais $\mathrm{x}=\left(\mathrm{x}_{1}, \mathrm{x}_{2}, \cdots, \mathrm{x}_{\mathrm{n}}\right)$, onde alguns não aparecem nenhuma vez, outros aparecem uma vez, outros aparecem duas vezes, três vezes etc (MURTEIRA, 1990). 
Da distribuição $\hat{\mathrm{F}}_{(\mathrm{n})}(\mathrm{x})$ obtêm-se B amostras bootstrap de mesmo tamanho n, como apresentada na sequência abaixo:

$$
\begin{aligned}
& \mathrm{x}_{1}^{*}=\left[\mathrm{x}_{11}^{*}, \mathrm{x}_{12}, \cdots, \mathrm{x}_{1 \mathrm{n}}^{*}\right]
\end{aligned}
$$

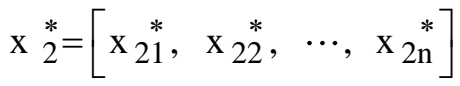

$$
\begin{aligned}
& \vdots \\
& \mathrm{x}_{\mathrm{B}}^{*}=\left[\mathrm{x}_{\mathrm{B} 1}^{*}, \mathrm{x}_{\mathrm{B} 2}^{*}, \cdots, \mathrm{x}_{\mathrm{Bn}}^{*}\right]
\end{aligned}
$$

Neste caso, o estimador do desvio padrão da população é calculado pela Equação 3.

$$
\hat{\mathrm{s}}_{\mathrm{B}}=\sqrt{\frac{1}{\mathrm{n}-1} \sum_{\mathrm{i}=1}^{\mathrm{n}}\left(\overline{\mathrm{x}}_{\mathrm{i}}-\overline{\mathrm{x}}_{\mathrm{B}}\right)^{2}}
$$

Especificamente, $\overline{\mathrm{x}}_{\mathrm{i}}$, pode ser substituído pelo estimador $\hat{\Theta}_{\mathrm{i}}$, para cada procedimento de reamostragem. A média $\overline{\mathrm{x}}_{\mathrm{B}}$ pode também ser substituída por $\hat{\Theta}_{\mathrm{B}}$, que é a média aritmética dos $\mathrm{n}$ estimadores bootstrap. A diferença $\hat{\Theta}_{B}-\hat{\Theta}_{i}$ é o estimador do enviesamento de $\hat{\Theta}$. Deste modo, o estimador do erro padrão de $\hat{\Theta}$ é calculado pela Equação 4 .

$$
\hat{\mathrm{s}}_{\mathrm{B}}=\sqrt{\frac{1}{\mathrm{~B}-1} \sum_{\mathrm{i}=1}^{\mathrm{B}}\left(\hat{\Theta}_{\mathrm{i}}-\hat{\Theta}_{\mathrm{B}}\right)^{2}}
$$

Onde: $\hat{\Theta}_{i}=\sum_{i=1}^{n} \frac{x_{i}^{*}}{n}$ e $\hat{\Theta}_{B}=\sum_{i=1}^{B} \frac{\hat{\Theta}_{i}}{B}$

A grande vantagem do método bootstrap é que ele pode ser aplicado a praticamente, qualquer estatística $\hat{\Theta}$, não se limitando apenas à média $\hat{\Theta}=\bar{x}$ (MURTEIRA, 1990). Isto é muito importante, uma vez que para algumas estatísticas ou não existem fórmulas analíticas ou, quando existem, são difíceis e aproximadas para a estimativa dos seus respectivos erros padrões. A reamostragem bootstrap tenta realizar o que seria desejável realizar na prática: repetir os procedimentos experimentais. A técnica de reamostragem bootstrap passa pelo algoritmo de Monte Carlo, onde, um dispositivo gerador de números aleatórios selecionou números inteiros $\mathrm{i}_{1}, \mathrm{i}_{2}, \cdots, \mathrm{i}_{\mathrm{n}}$, cada um dos quais é igual a algum valor entre $1 \mathrm{e}$ n com probabilidade $\frac{1}{n}$. A amostra formada consiste dos correspondentes elementos do conjunto original $\mathrm{x}=\left(\mathrm{x}_{1}, \mathrm{x}_{2}, \cdots, \mathrm{x}_{\mathrm{n}}\right)$. Na prática, constrói-se a distribuição bootstrap de $\mathrm{F}$ por Monte Carlo com um número de repetições, $\mathrm{B}$, suficientemente grande. Um indicador do tamanho adequado de $\mathrm{B}$, independente do custo computacional, é a qualidade da convergência da estimativa do parâmetro para a estimativa natural do parâmetro $\hat{\Theta}_{\mathrm{B}}(\mathrm{B} \rightarrow \infty) \rightarrow \Theta(\mathrm{F})$, sendo a construção do algoritmo geralmente simples (MURTEIRA, 1990). Sua convergência está garantida pela lei dos Grandes Números, pois, $\left(x_{1}^{*}, x_{2}^{*}, \cdots, x_{n}^{*}\right)$ nada mais são do que uma amostra de variáveis aleatórias independentes e identicamente distribuídas com distribuição condicional de $\hat{\Theta}_{\mathrm{B}}$. Assim, quando B tende a infinito, a média amostral $\widehat{\Theta}_{\mathrm{B}}$ aproxima-se de $\Theta(\mathrm{EFRON}, 1982)$. O seguinte algoritmo foi construído pelo método de Monte Carlo para determinar a distribuição bootstrap: 
(1) Da amostra experimental, sorteou-se, utilizando um gerador de números aleatórios com probabilidade $\frac{1}{\mathrm{n}}$, os $\mathrm{n}$ valores com reposição para formar as amostras bootstrap de mesmo tamanho da original.

(2) Computou-se a média aritmética em cada procedimento de reamostragem.

(3) Repetiu-se o passo (2) um número B de vezes obtendo, dessa maneira, B valores da estatística em questão.

(4) Obtiveram-se as B médias para formar a distribuição $\hat{F}$.

(5) Determinou-se o estimador $\hat{\Theta}_{B}$ da distribuição $\hat{F}$.

$\mathrm{O}$ valor de $\hat{\Theta}_{\mathrm{B}}$ foi utilizado como a média da concentração do ${ }^{210} \mathrm{~Pb}$. O procedimento de simulação foi realizado utilizando um programa desenvolvido na linguagem $\mathrm{C}++$, com o gerador de números aleatórios renu (VIEIRA, 2004). A Figura 1 ilustra a operação do programa para construir a distribuição bootstrap pelo algoritmo de Monte Carlo. No presente trabalho, as iterações bootstrap foram realizadas de acordo com o tamanho e a variação nos resultados. Algumas considerações sobre a consistência desse método foram discutidas por Bickel e Freedman (1981). Os conceitos básicos, propriedades teóricas e aplicações podem ser encontrados em Efron e Tibshirani (1993). O método bootstrap diminui significativamente, a influência dos outiliers num conjunto de dados radioecológicos (SILVA et al., 2007; SILVA et al., 2015). Portanto, dentro desse contexto, o método bootstrap constituise numa ferramenta estatisticamente robusta para construir intervalos de confiança na determinação do limite de detecção em radioquímica.

Figura 1 - Esquema do algoritmo de Monte Carlo para construir a distribuição bootstrap

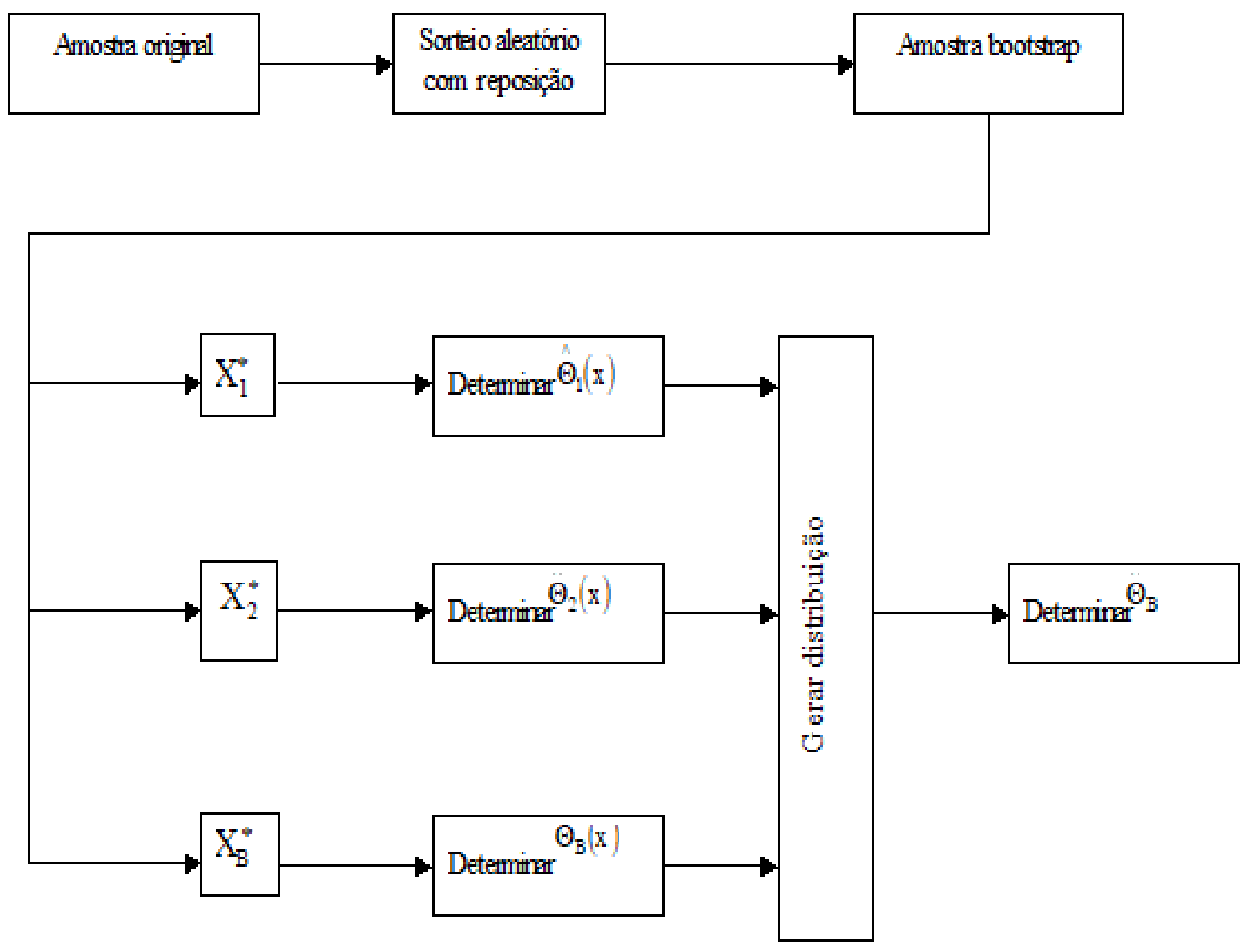




\section{RESULTADO E DISCUSSÃO}

\section{Avaliação do método de análise do ${ }^{210} \mathrm{~Pb}$}

Os valores médios da precisão e da exatidão foram respectivamente, 3,5\% e 96\%, respectivamente. O rendimento químico médio da técnica utilizada foi de $97 \%$. Esse resultado indica a grande adaptabilidade e a aplicabilidade do método da troca iônica na quantificação do ${ }^{210} \mathrm{~Pb}$ nas amostras analisadas. Entretanto, segundo Jia e Torri (2007), a exatidão do resultado final é afetada diretamente pela pureza das fontes de ${ }^{210} \mathrm{~Pb}$. De acordo com esses autores, os radionuclídeos emissores beta das séries radioativas naturais do urânio e de tório interferem grandemente na quantificação do ${ }^{210} \mathrm{~Pb}$ em análise de amostras ambientais. Por outro lado, segundo, Godoy et al. (1998) e Jia e Torri (2007), o método da resina trocadora de íons, constitui-se um procedimento altamente eficaz para separar o ${ }^{210} \mathrm{~Pb}$ de outros emissores beta em amostras ambientais. Decorrente disso, no presente trabalho, foi verificado nas amostras padrões, preparadas conforme item 2.2, o grau de separação do ${ }^{210} \mathrm{~Pb}$ dos demais emissores beta. Para tanto, observou-se o crescimento radioativo do ${ }^{210} \mathrm{Bi}$ nas amostras padrões. As Figuras 3, 4, 5 e 6 mostram o crescimento do ${ }^{210} \mathrm{Bi}$ nas amostras de água deionizada, nas quais foram adicionados padrões de ${ }^{210} \mathrm{~Pb}$ com concentrações iguais a $3 \mathrm{~Bq}$.

Nas Figuras 2, 3, 4 e 5, observa-se que, quando os valores experimentais e teóricos tendem ao valor do equilíbrio secular radioativo, a curva experimental e teórica coincidem. Nesse caso, o equilíbrio secular entre o ${ }^{210} \mathrm{~Pb}$ e o ${ }^{210} \mathrm{Bi}$ foi alcançado num tempo de aproximadamente, 50.000 minutos ( $\cong 35$ dias), após o procedimento de eluição do ${ }^{210} \mathrm{~Pb}$ da resina trocadora de íons. Sendo assim, foi considerada desprezível, a interferência dos demais emissores beta existentes nas amostras analisadas. O tempo de equilíbrio secular calculado no presente trabalho, foi aproximadamente igual, àqueles encontrados por Jia e Torri (2007) e Silva et al. (2009), em análises de amostras de rochas e solos.

Figura 2 - Tempo de crescimento do ${ }^{210} \mathrm{Bi}$ na amostra padrão 1 após a eluição

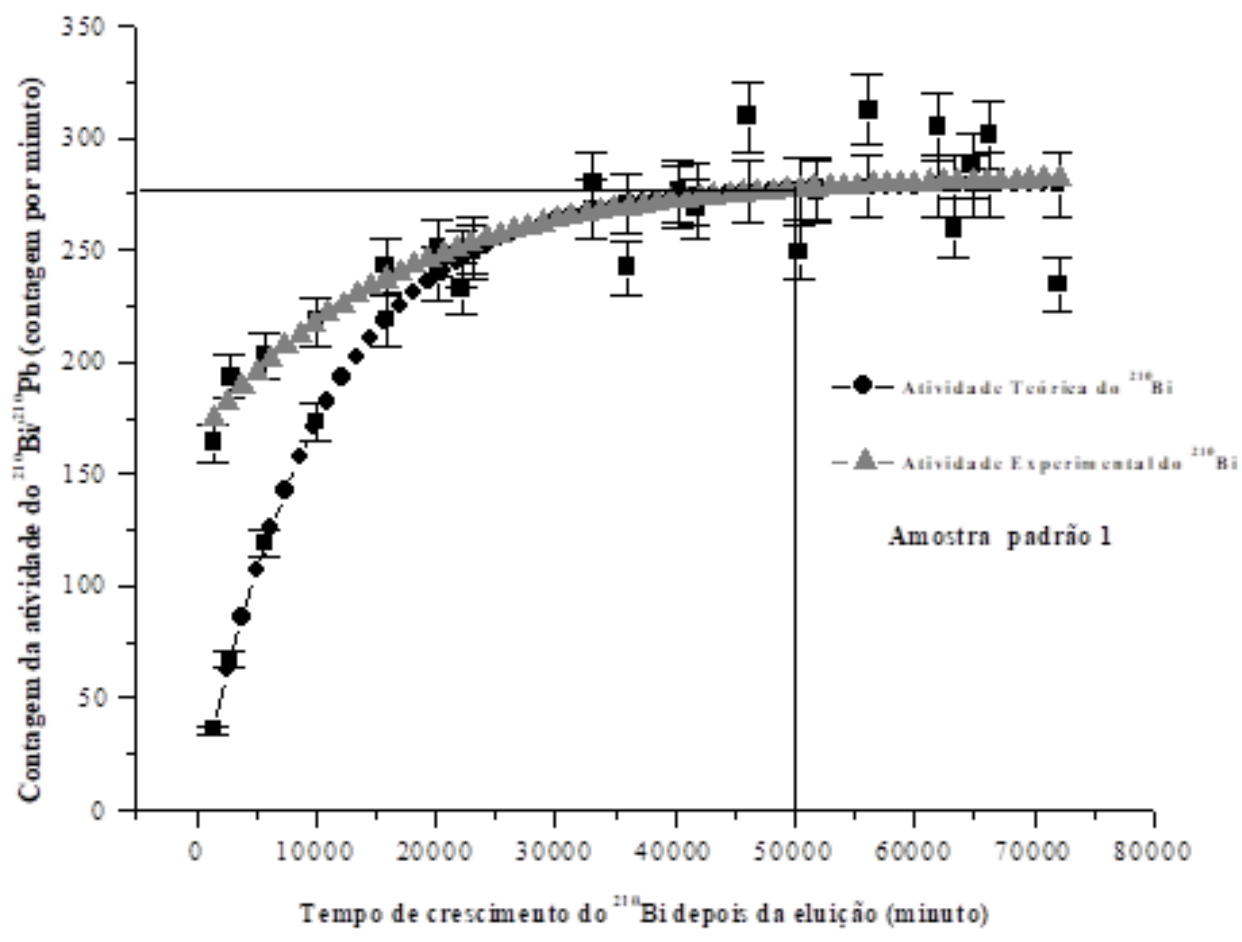


Figura 3 - Tempo de crescimento do ${ }^{210} \mathrm{Bi}$ na amostra padrão 2 após a eluição

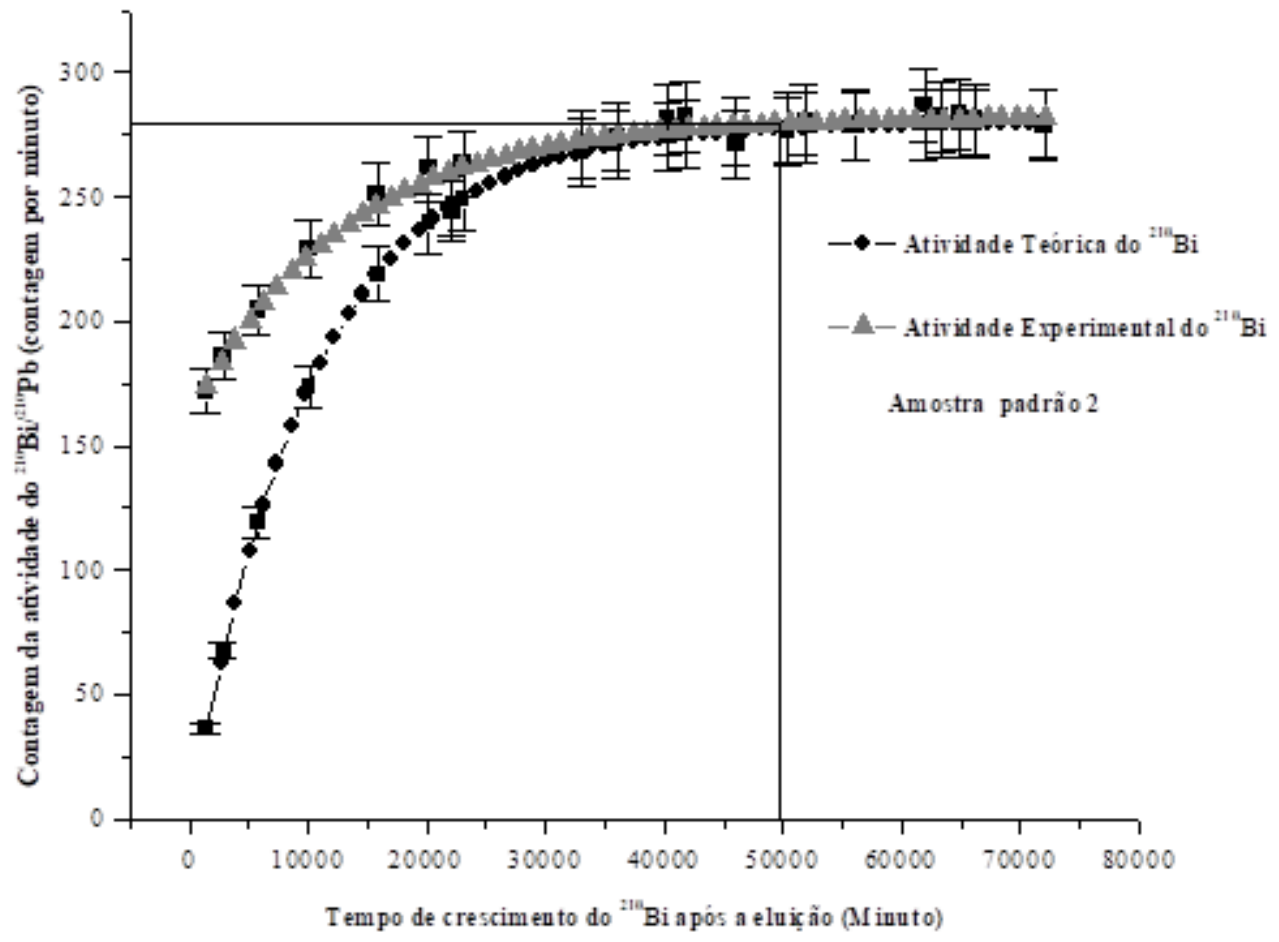

Figura 4 - Tempo de crescimento do ${ }^{210} \mathrm{Bi}$ na amostra padrão 3 após a eluição

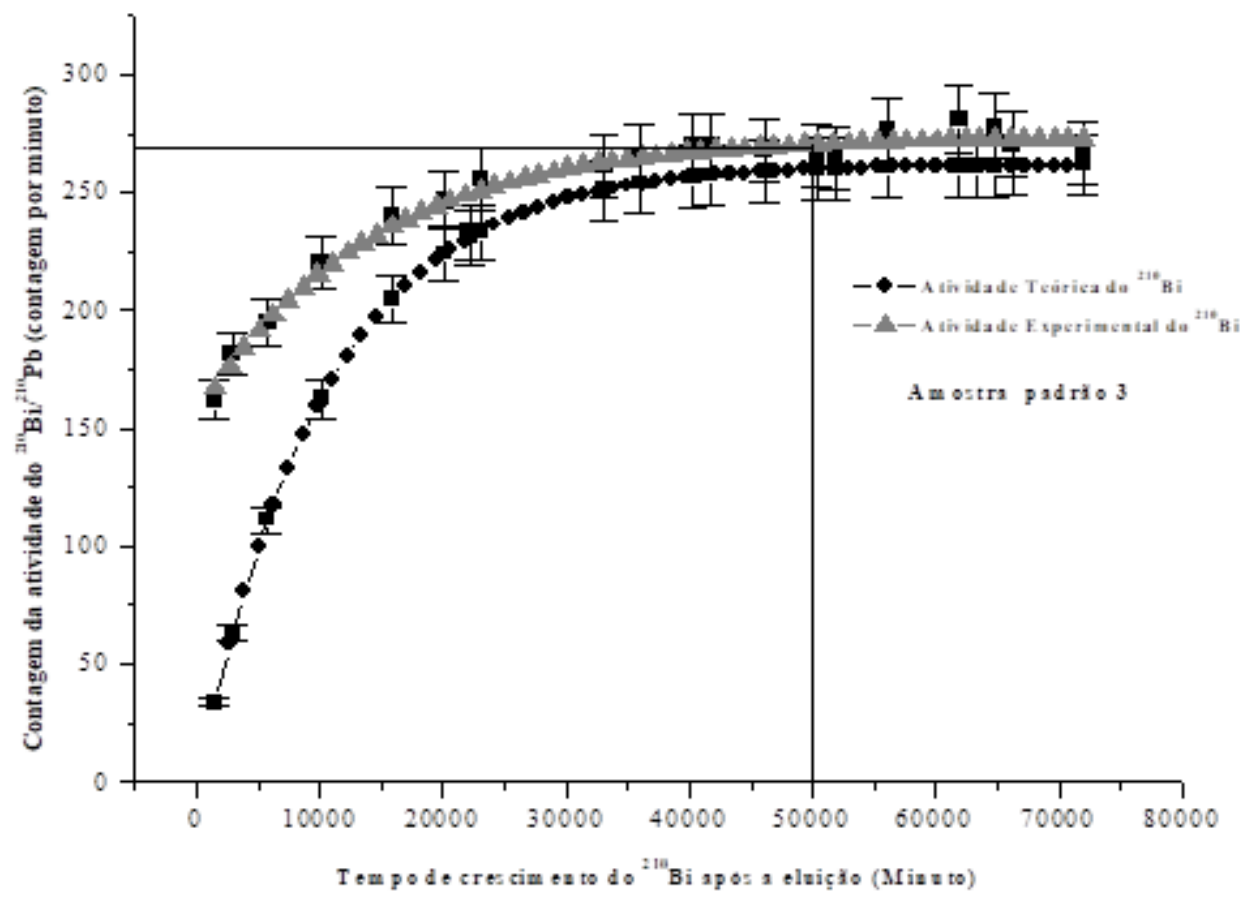


Figura 5 - Tempo de crescimento do ${ }^{210} \mathrm{Bi}$ na amostra padrão 4 após a eluição

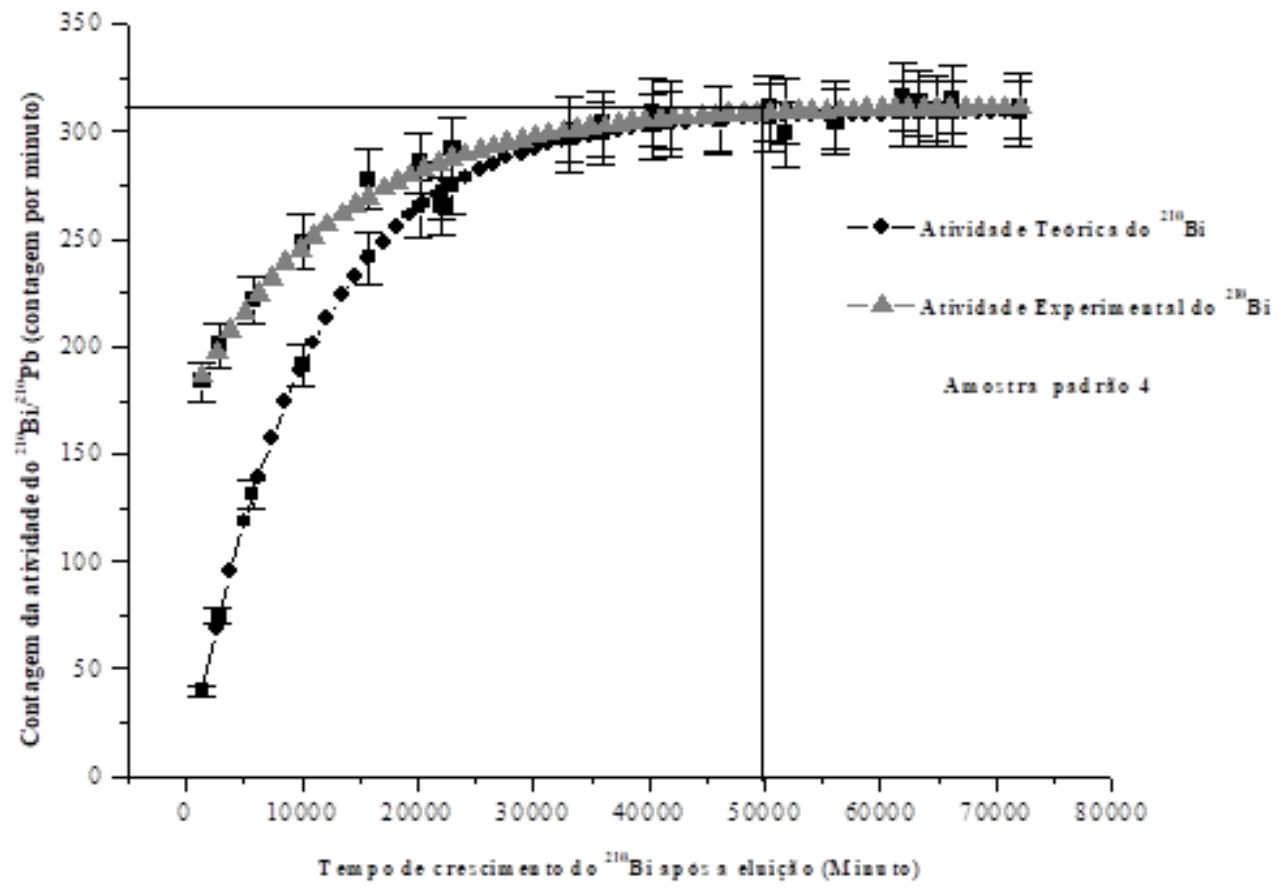

\section{Calibração do detector para análise do ${ }^{210} \mathrm{~Pb}$}

Nos procedimentos de calibração do detector Canberra Tennelec S5E, prepararam-se amostras padrões conformes procedimentos apresentados no item anterior. As concentrações dos padrões variaram de 0,153 a 6,124 Bq em análise de quadruplicatas. A Figura 6 mostra a curva de calibração média obtidas de cinco análises de concentrações diferentes. A eficiência de contagem no platô beta $(\eta)$ foi determinada diretamente na Figura 6, pois ela é numericamente igual ao coeficiente angular da reta. $O$ valor de $\eta$ foi de $19,7 \mathrm{cpm} . \mathrm{Bq}^{-1}$. Isso resultou numa eficiência de contagem beta de aproximadamente, $33 \%$.

Figura 6 - Curva de calibração média do detector Canberra Tennelec S5E no platô beta

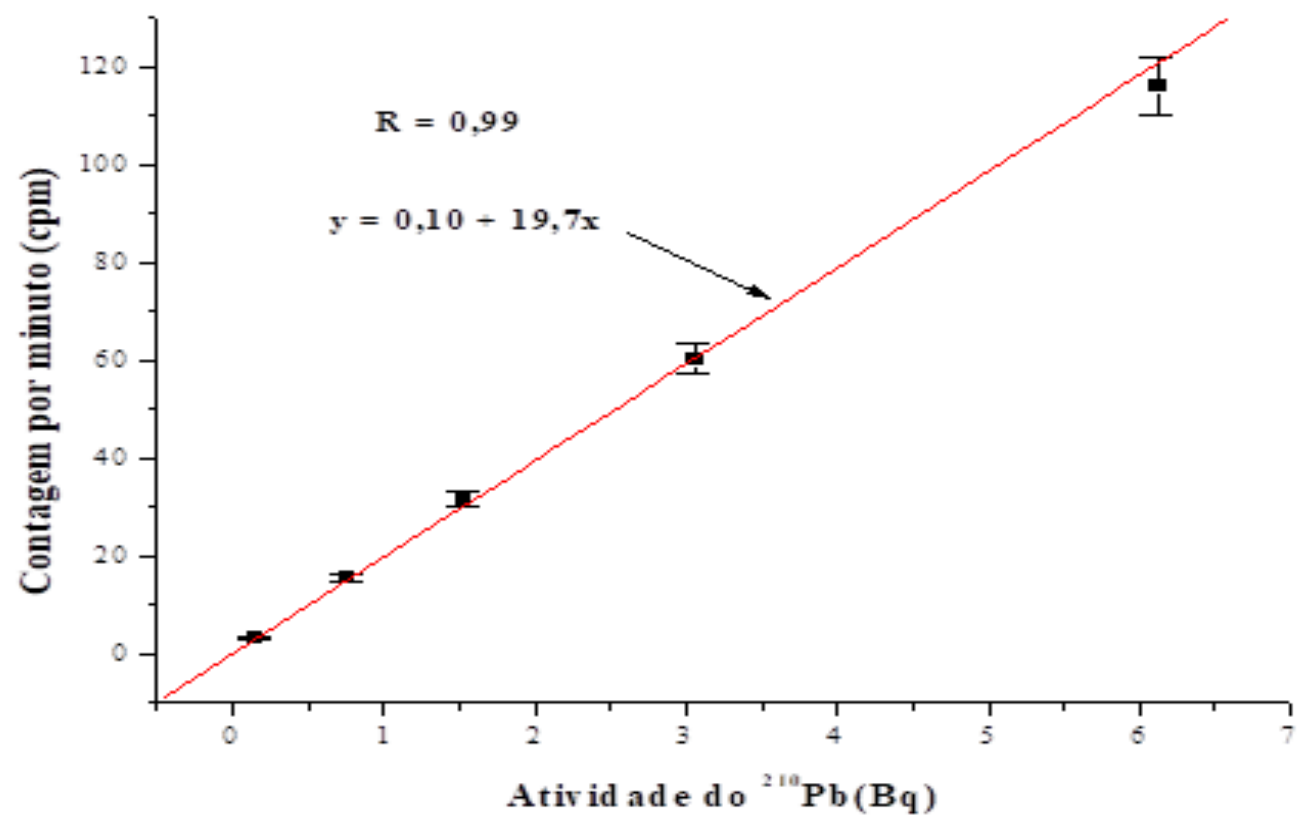




\section{Concentração de ${ }^{210} \mathrm{~Pb}$ nas amostras de água deionizada}

As concentrações líquidas absolutas do ${ }^{210} \mathrm{~Pb}$ nas amostras de água deionizada encontram-se apresentadas na Tabela 1. Estes resultados foram obtidos subtraindo-se a contagem da radiação beta do precipitado e a contagem beta do papel milipore sem o precipitado. Observar-se na Tabela 1 grande variabilidade nos valores das concentrações de ${ }^{210} \mathrm{~Pb}$. Medições de radioatividade ambiental pode envolver material que contenha quantidades muito pequenas do radionuclídeo de interesse, como apresentado na Tabela 1. A incerteza de medição muitas vezes torna difícil distinguir tais pequenas quantidades de zero. Assim, uma importante característica do desempenho de um processo analítico de medição é a sua capacidade de deteç̧ão, que é normalmente expressa como a menor concentração de analito (limite de detecção) que pode ser distinguido com segurança a partir de zero. Em radioquímica, muitas vezes, o limite de detecção é obtido apenas das contagens do instrumento de fontes livres de analito. Entretanto, em princípio, o limite de detecção deve ser estimado usando as contagens obtidas de amostras livres de analito que foram submetidas aos mesmos procedimentos experimentais das amostras de interesse (Tabela 1). Este procedimento dever ser realizado para verificar o quanto o método utilizado afeta o processamento das amostras antes da análise instrumental. Por outro lado, planejamento eficaz em radioquímica não requer apenas conhecimento das capacidades de detecção dos procedimentos analíticos que será ou poderá ser usado, mas também, do tipo de modelo estatístico que será utilizado na estimativa da concentração mínima detectável. Em qualquer processo de análise em laboratório, a pergunta óbvia a ser respondida é: será que a amostra contém quantidade positiva do analito? Geralmente, a incerteza do valor medido torna a pergunta difícil de responder. Mas, existem diferentes métodos estatísticos que são utilizados na tomada de decisão na estimativa da concentração mínima detectável. Os métodos mais frequentemente utilizados em radioquímica envolvem os princípios da estatística de testes de hipóteses.

Tabela 1. Concentração absoluta de ${ }^{210} \mathrm{~Pb}$ nas amostras de água deionizada.

\begin{tabular}{cc}
\hline Código da amostra & Concentração de ${ }^{\mathbf{2 1 0}} \mathbf{P b}\left(\mathbf{m B q} \cdot \mathbf{L}^{\mathbf{1}}\right)$ \\
\hline Amostra 1 & 12 \\
Amostra 2 & 25 \\
Amostra 3 & 13 \\
Amostra 4 & 12 \\
Amostra 5 & 52 \\
Amostra 6 & 12 \\
Amostra 7 & 44 \\
Amostra 8 & 22 \\
Amostra 9 & 60 \\
Amostra 10 & 49 \\
Amostra 11 & 69 \\
\hline
\end{tabular}

Estatisticamente, detectar a presença de um analito numa amostra de laboratório, com apresentado na Tabela 1, com base nos dados de medição, significa escolher entre duas hipóteses sobre a amostra. A primeira hipótese é a hipótese nula $\mathrm{H}_{0}$ : a amostra é livre de analito. A segunda hipótese é a hipótese 
alternativa $\mathrm{H}_{1}$ : a amostra não é livre de analito. A hipótese nula $\mathrm{H}_{0}$ representa o status quo, ou seja, a circunstância que está sendo testada, e o objetivo dos testes de hipóteses é sempre tentar rejeitar a hipótese nula. A hipótese alternativa $\mathrm{H}_{1}$ representa o que se deseja provar ou estabelecer, sendo formulada para contradizer a hipótese nula. Em radioquímica, presume-se que a hipótese nula é verdadeira, a menos que haja provas estatísticas suficientes para rejeitá-la. Se a evidência é forte o suficiente, a hipótese nula é rejeitada em favor da hipótese alternativa. São dois os tipos de erros que podemos cometer na realização de um teste de hipóteses: (1) rejeitar a hipótese $\mathrm{H}_{0}$, quando ela é verdadeira e (2) não rejeitar a hipótese $\mathrm{H}_{0}$, quando ela é falsa (MONTGOMERY, 2009). Se a hipótese $\mathrm{H}_{0}$ for verdadeira e não rejeitada ou falsa e rejeitada, a decisão estará correta.

No entanto, se a hipótese $\mathrm{H}_{0}$ for rejeitada sendo verdadeira, ou se não for rejeitada sendo falsa, a decisão estará errada. O primeiro destes erros é chamado de Erro do Tipo I e a probabilidade de cometê-lo é denotada pela letra grega $\alpha$ (alfa). O segundo é chamado de Erro do Tipo II e a probabilidade de cometêlo é denotada pela letra grega $\beta$ (beta). Assim temos, $\alpha=\mathrm{p}($ Erro tipo 1$)=\mathrm{p}\left(\right.$ rejeitar $\mathrm{H}_{0}$ dado $\mathrm{H}_{0}$ verdadeira $) \mathrm{e}$ $\beta=\mathrm{p}($ Erro tipo 2$)=\mathrm{p}\left(\right.$ aceitar $\mathrm{H}_{0}$ dado $\mathrm{H}_{0}$ falsa $)$. Consideremos um teste unilateral dado pelas hipóteses: $\left\{\begin{array}{l}\mathrm{H}_{0}: \mu_{0}=\mu \\ \mathrm{H}_{1}: \mu<\mu_{0}\end{array}\right.$. Neste caso, a região de rejeição é determinada por $\overline{\mathrm{X}}<\mathrm{X}_{\mathrm{C}}$ e a interpretação dos erros pode ser vista como: $\alpha=\mathrm{p}\left(\overline{\mathrm{X}}<\mathrm{X}_{\mathrm{C}} \mid \mu=\mu_{0}\right)$ e $\beta=\mathrm{p}\left(\overline{\mathrm{X}}>\mathrm{X}_{\mathrm{C}} \mid \mu<\mu_{0}\right)$. Neste caso, $\overline{\mathrm{X}}$ e $\mathrm{X}_{\mathrm{C}}$ são respectivamente, a média aritmética e o valor crítico. A situação ideal é aquela em que ambas as probabilidades, $\alpha$ e $\beta$, são próximas de zero. No entanto, é fácil ver que, à medida que diminuímos $\alpha, \beta$ aumenta. A Figura 7 apresenta esta relação (MONTGOMERY, 2009).

Figura 7 - Representação geométrica dos erros de hipóteses

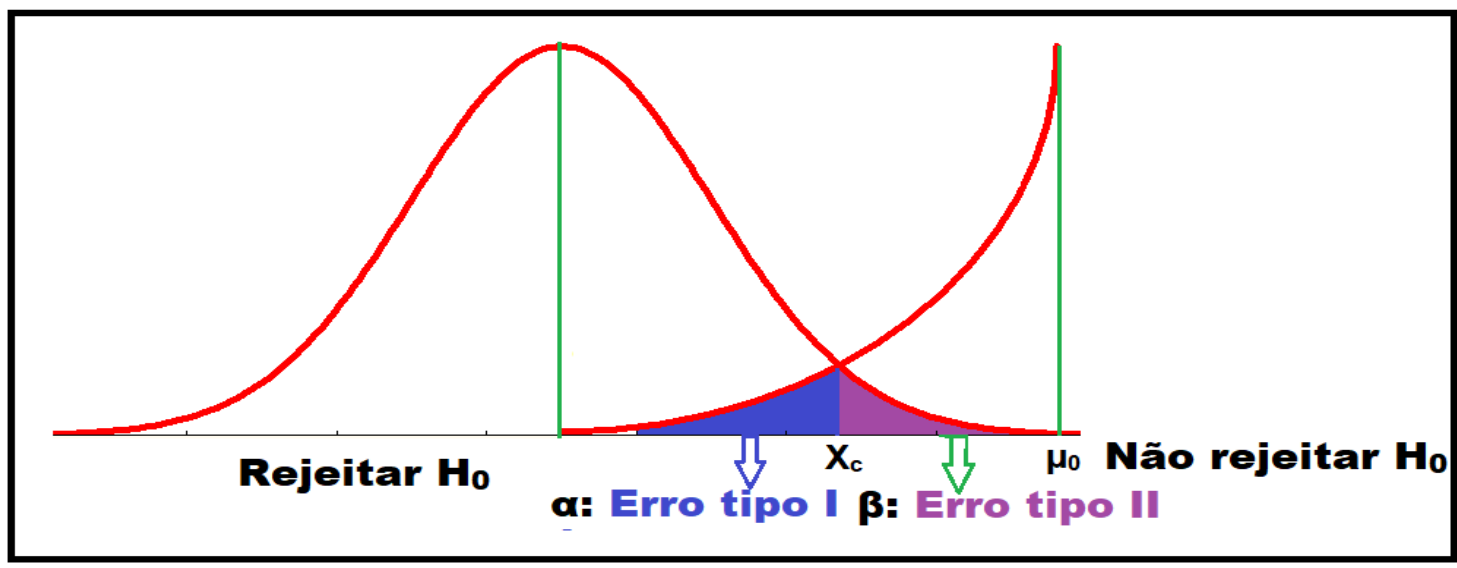

Para um teste de hipóteses do tipo $\left\{\begin{array}{l}\mathrm{H}_{0}: \mu=\mu_{0} \\ \mathrm{H}_{1}: \mu<\mu_{0}\end{array}\right.$, onde estamos interessados em testar a média de uma população, utilizamos a Equação 5.

$$
\mathrm{Z}=\frac{\overline{\mathrm{X}}-\mu_{0}}{\frac{\sigma}{\sqrt{\mathrm{n}}}}
$$


A partir do Teorema Central do Limite, sabemos que, desde que tenhamos um tamanho amostral suficientemente grande, esta estatística tem distribuição normal padrão, isto é, $Z \square N(0,1)$. Com os valores de $\mathrm{Z}$ e da especificação do erro cometido, podemos definir a região crítica do teste. Vamos considerar que o erro mais importante a ser evitado seja o Erro do Tipo I. A probabilidade de ocorrer o Erro do tipo I $(\alpha)$ é denominada nível de significância do teste. O complementar do nível de significância $(1-\alpha)$ é denominado nível de confiança. Supondo que o nível de significância $\alpha$ seja conhecido, temos condições de determinar o valor crítico. Se considerarmos o teste bilateral $\left\{\begin{array}{l}\mathrm{H}_{0}: \mu=\mu_{0} \\ \mathrm{H}_{1}: \mu \neq \mu_{0}\end{array}\right.$, a Figura 8 representa a região de rejeição para um valor fixo de $\alpha$ (MONTGOMERY, 2009).

Figura 8 - Região de rejeição do teste bilateral para um valor fixo de $\alpha$

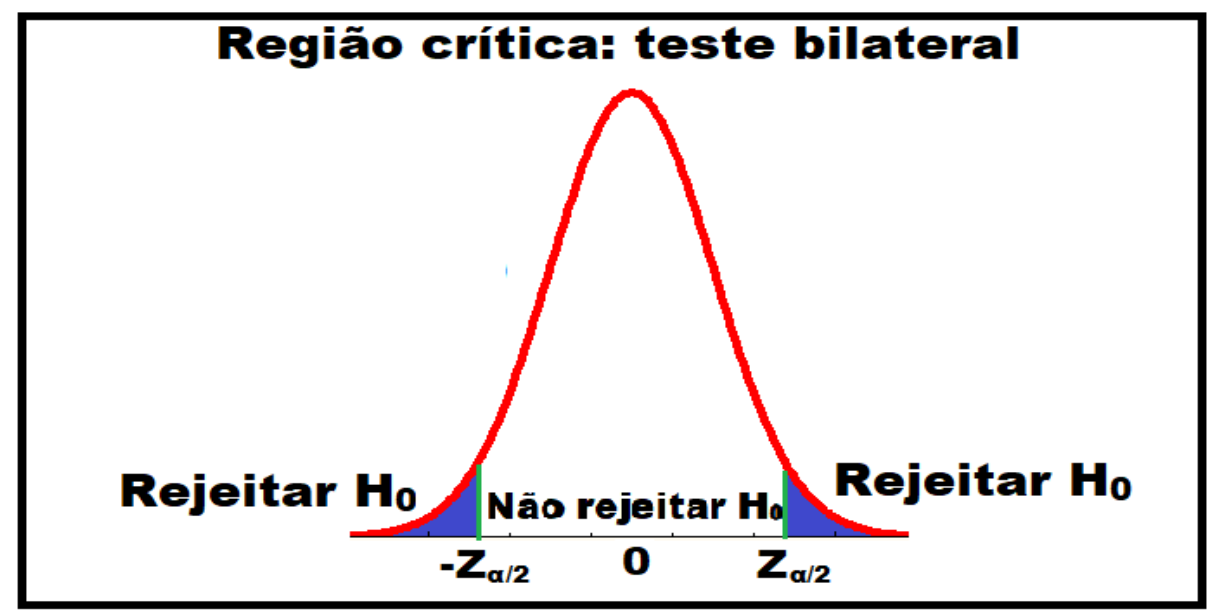

Considerando um teste lateral à direita $\left\{\begin{array}{l}\mathrm{H}_{0}: \mu=\mu_{0} \\ \mathrm{H}_{1}: \mu>\mu_{0}\end{array}\right.$, a região crítica é representada pela Figura 9. E, se considerarmos o teste unilateral à esquerda $\left\{\begin{array}{l}\mathrm{H}_{0}: \mu=\mu_{0} \\ \mathrm{H}_{1}: \mu<\mu_{0}\end{array}\right.$, a região crítica é representada pela Figura 10 (MONTGOMERY, 2009).

Figura 9 - Região de rejeição do teste unilateral à direita para um valor fixo de $\alpha$

\section{Região crítica: teste unilateral à direita}

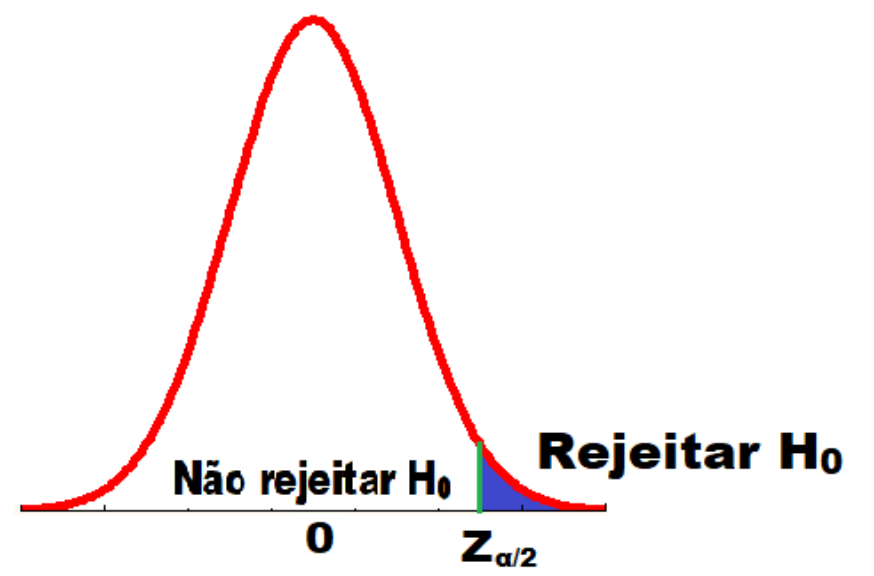


Figura 10 - Região de rejeição do teste unilateral à esquerda para um valor fixo de $\alpha$

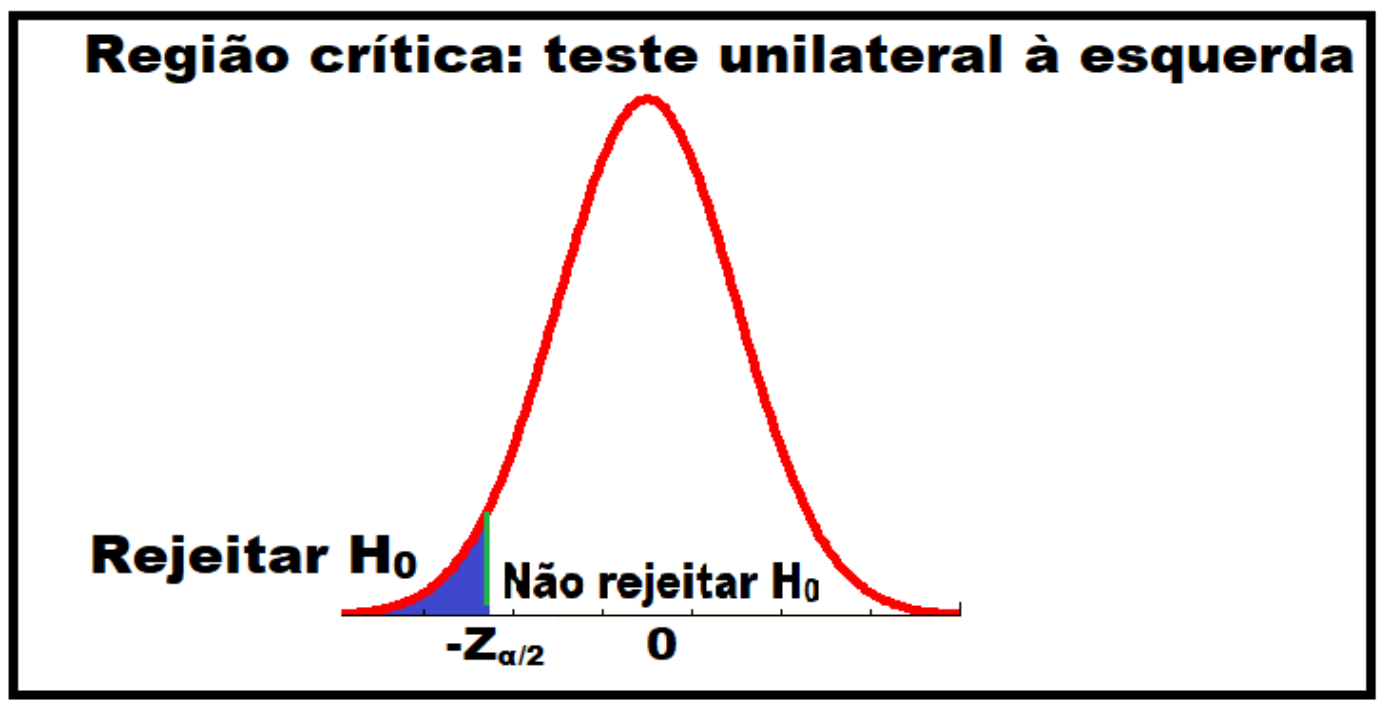

Os valores $-Z_{\alpha}$ e $Z_{\alpha}$ nas Figuras 9 e 10 são tais que as áreas à esquerda e à direita, respectivamente, sob a curva normal padrão, valem $\alpha$. Agora, os valores $-Z_{\alpha / 2}$ e $Z_{\alpha / 2}$, na Figura 8 , são tais que as áreas à esquerda e à direita, respectivamente, sob a curva normal padrão, valem $\alpha / 2$. Como já foi dito, o objetivo do teste de hipótese é determinar, através de uma estatística, se a hipótese nula é aceitável ou não. Essa decisão é tomada considerando a região de rejeição ou região crítica $\left(\mathrm{X}_{\mathrm{C}}\right)$. Caso o valor observado da estatística pertença à região de rejeição, rejeitamos $\mathrm{H}_{0}$; caso contrário, não rejeitamos $\mathrm{H}_{0}$. Analogamente, definimos a região de aceitação (complementar da região de rejeição): caso o valor observado pertença à região de aceitação, não rejeitamos $\mathrm{H}_{0}$; se não pertencer, rejeitamos. Se o nível de significância é 0,05 , os valores críticos são-1,645 ou 1,645 para as alternativas unilaterais e -1,96 e 1,96 para a alternativa bilateral; se o nível de significância é 0,01 , os valores críticos são $-2,33$ ou 2,33 para as alternativas unilaterais e $-2,575$ e 2,575 para a alternativa bilateral (valores obtidos na Tabela da distribuição normal) (MONTGOMERY, 2009).

A utilização de testes de hipóteses para decidir se um analito está presente numa amostra, como aquelas apresentadas na Tabela 1, é conceitualmente simples, mas o tema ainda gera confusão e discordância entre os muitos pesquisadores. Para Altshuler e Pasternack (1963), Nicholson (1963 e 1968), numa análise radioquímica, o limite de detecção verdadeiro é definido como a atividade mínima detectável. Comentando sobre a definição de Altshuler e Pasternack (1963), Currie (1968) afirmou que: "o significado desta forma particular de definição é que ela permite determinar, para um dado processo de medição, o menor sinal verdadeiro que vai ser detectado com nível de confiança de 1- $\beta$, onde, o mecanismo de uma decisão a posteriori foi construído com o nível de significância $\alpha$ (concluindo falsamente que uma observação em branco representa um sinal real). Sinais verdadeiros encontrados entre zero e o valor crítico terá valores maiores para $\beta$, e, portanto, apesar de poderem ser "detectado", essa detecção não pode ser considerada confiável". Por outro lado, para Currie (1968), o limite de detecção é definido como o sinal resultante a partir de uma amostra de background (branco) que é idêntica, em princípio, a amostra de interesse, exceto que a substância procurada está ausente, ou é tão pequena que não pode ser detectada. Isto mostra a importância de se ter procedimentos padrões estabelecidos na metodologia de análise, tais como foram usados na determinação do ${ }^{210} \mathrm{~Pb}$ nas amostras apresentadas na Tabela 1.

Segundo Currie (1968), o valor máximo aceitável para $\alpha$, num conjunto de dados de amostras (branco) estabelece o valor crítico, Xc, sobre a qual pode ser tomada decisões. Considerando a Figura 1, 
operacionalmente, um sinal observado deve exceder o valor crítico Xc para produzir a decisão, "detectado". Assim, a distribuição de probabilidade de resultados possíveis, quando o verdadeiro sinal (líquido) é zero, intersecta Xc de modo que a fracção, 1- $\alpha$, corresponde à decisão (correta), "não detectado". Uma vez que o valor crítico tenha sido definido, um limite de detecção a priori, pode ser estabelecido, especificando Xc no nível aceitável, $\beta$, para o Erro do Tipo II, o que caracteriza a distribuição de probabilidade de a rede sinalizar quando o seu verdadeiro valor limite médio é igual ao limite de detecção. Matematicamente, Currie (1968) determinou o valor do limite de detecção $\left(\mathrm{L}_{\mathrm{D}}\right)$ usando a Equação 6.

$$
\mathrm{L}_{\mathrm{D}}=\mathrm{L}_{\mathrm{C}}+\mathrm{k} \sigma
$$

Onde: Lc é o limite de detecção crítico; $\mathrm{k}$ é a constante de probabilidade para 1- $\beta$ e $\sigma^{2}=2 \sigma_{\mathrm{B}}^{2}$, sendo $\sigma_{\mathrm{B}}^{2}$ a variância dos valores das amostra de background (branco). Em alguns casos, $\sigma^{2}$ é igual a variância das medidas dos sinais observados. Fazendo modificação na Figura 7 e substituindo Xc por Lc e $\mu_{0}$ por $L_{D}$, obtemos a Figura 11, conforme definição de Currie (1968). Este autor, definiu $L_{C}$ como sendo o nível do sinal líquido (resposta do instrumento) acima do qual um sinal observado $\mathrm{S}$ pode ser reconhecido de forma confiável como "detectado" e $\mathrm{L}_{\mathrm{D}}$ como sinal líquido "verdadeiro" que pode a priori, levar a detecção.

Em estudos radioquímicos, tanto os dados obtidos das amostras background (branco) como as amostras de interesse, consideram-se possuírem distribuição de Poisson. Caso, o conjunto de dados sejam suficientemente grande, a distribuição de Poisson tende para a distribuição Normal. Sendo assim, pode-se facilmente estimar a variação do sinal de rede e estabelecer níveis aproximados de confiança e significância. Matematicamente, o limite crítico $\left(\mathrm{L}_{\mathrm{C}}\right)$ é calculado pela Equação 7 (CURRIE, 1968).

$$
\mathrm{L}_{\mathrm{C}}=\mathrm{k} \sigma
$$

Figura 11 - Limite crítico Lc e limite de detecção $L_{D}$

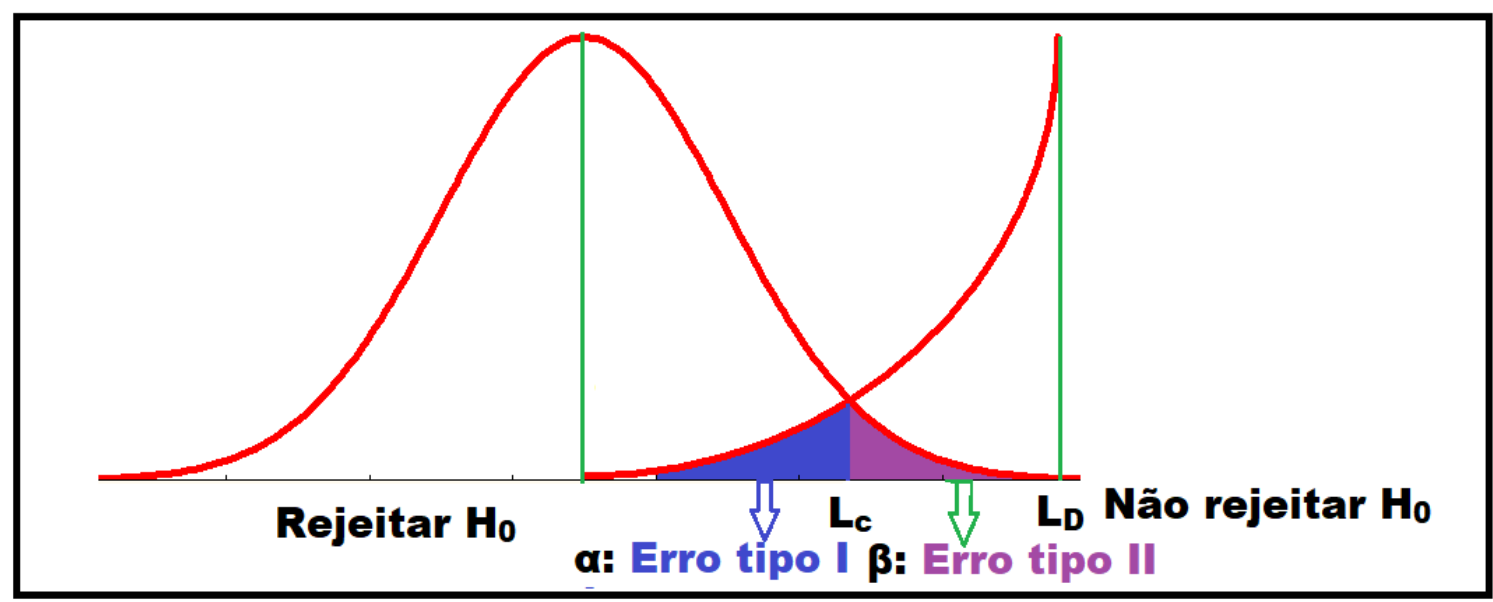

Currie (1968) demonstrou que $\mathrm{L}_{\mathrm{D}}=2 \mathrm{~L}_{\mathrm{C}}$. Segundo este autor, isto acontece na maioria dos casos. Partindo do princípio de que o risco aceitável é de 5\%, e que os erros aleatórios são normalmente distribuídos, a constante k, assume o valor de 1,645. Porém, Currie (1968) determinou uma expressão geral para calcular o limite de detecção, conforme mostra a Equação 8.

$$
\mathrm{L}_{\mathrm{D}}=\mathrm{k}^{2}+2 \mathrm{~L}_{\mathrm{C}}
$$

$\mathrm{Na}$ estimativa do limite de deteç̧ão $\mathrm{L}_{\mathrm{D}}$ existe a necessidade de fazer mais do que comparar um resultado experimental com o nível de decisão, a fim de chegar a uma conclusão qualitativa. Entretanto, na maioria dos casos tem-se disponível uma estimativa do sinal líquido $\mathrm{S}$, em relação ao seu desvio padrão $\sigma$. 
Para tanto, é necessário calcular o intervalo de confiança no qual o sinal líquido verdadeiro $\mathrm{L}_{\mathrm{D}}$, certamente ocorrerá. Neste caso, o intervalo de confiança é estimado utilizando $Z_{1-\frac{\gamma}{2}} \sigma$ e o correspondente nível de confiança de $1-\gamma$. O intervalo de confiança é construído baseado na distribuição normal padronizada, com Prob $\left\{\mathrm{Z} \leq \mathrm{Z}_{1-\frac{\gamma}{2}}\right\}=1-\frac{\gamma}{2}$. Porém, se o sinal de rede $\mathrm{S}$ e seu desvio-padrão são calculados por meio de replicação, então, substitui-se $S$ por $\bar{S}, \quad \sigma$ por $\frac{\mathrm{s}}{\sqrt{\mathrm{n}}}$ e $\mathrm{Z}_{1-\frac{\gamma}{2}} \sigma$ por $\mathrm{t}_{1-\frac{\gamma}{2}} \frac{\mathrm{s}}{\sqrt{\mathrm{n}}}$, onde s é o desvio padrão computado das $\mathrm{n}$ observações e $\mathrm{t}_{1-\frac{\gamma}{2}}$ representa $\mathrm{o}$ valor crítico da distribuição $\mathrm{t}-\mathrm{Student}$ correspondente a $\mathrm{n}-1$ grau de liberdade. Se $\mathrm{S}($ ou $\overline{\mathrm{S}})>\mathrm{L}_{\mathrm{C}}$, então, a decisão, "detectado", devem ser relatado, e deve ser dado um intervalo de confiança simétrico: $\overline{L_{D}} \pm Z_{1-\frac{\gamma}{2}} \frac{s}{\sqrt{n}}$ ou $\overline{L_{D}} \pm t_{1-\frac{\gamma}{2}} \frac{s}{\sqrt{n}}$. Caso, $\mathrm{S}($ ou $\overline{\mathrm{S}}) \leq \mathrm{L}_{\mathrm{C}}$, então, a decisão, "não detectado" é relatado, e deve ser dado um limite superior: $\overline{\mathrm{L}_{\mathrm{D}}}+\mathrm{Z}_{1-\gamma} \frac{\mathrm{s}}{\sqrt{\mathrm{n}}}$ ou $\overline{\mathrm{L}_{\mathrm{D}}}+\mathrm{t}_{1-\gamma} \frac{\mathrm{s}}{\sqrt{\mathrm{n}}}$, onde $\mathrm{Z}_{1-\gamma}$ e $\mathrm{t}_{1-\gamma}$ referem-se ao intervalo unilateral. No caso especial onde $\sigma$ é igual ao desvio padrão das amostras (branco) e $\mathrm{Z}_{1-\gamma}$ é utilizado, então, $\mathrm{L}_{\mathrm{C}}$ é numericamente igual a $\mathrm{Z}_{1-\gamma} \sigma$. Geralmente, isto leva à prática comum, de se querer igualar erroneamente, $Z_{1-\gamma} \sigma$ ao limite de deteç̧ão (com nível de confiança de 95\%). Tal situação, as vezes é inadequada, pois, o limite de deteç̧ão não pode ser estimado por um único nível de confiança, tendo apenas o valor do limite superior. Sendo assim, jamais podemos afirmar que um dado limite de detecção que foi calculado apenas com usando o limite superior, tem confiança de 1- $\beta$, pois, neste caso, o limite inferior não existe.

Às vezes, acontece de o limite superior ser confundido com o limite de detecção. Isto somente acontece quando os dois coincidem. Neste caso, o sinal líquido passar a ser $\mathrm{L}_{\mathrm{C}}, \sigma$ é o desvio padrão das amostras de background (branco) e o intervalo de confiança utilizado é unilateral. Tal coincidência não é acidental, pois o limite de detecção é, por definição, o limite superior máximo. Entretanto, deve ser lembrado que, em geral, o limite superior depende do resultado experimental específico, $\mathrm{S}$, enquanto que, o limite de detecção é independente de $\mathrm{S}$, dependendo, apenas do próprio processo de medição. Finalmente, a diferença entre $Z_{1-\frac{\gamma}{2}} \sigma$ e $\overline{L_{D}}+t_{1-\gamma} \frac{s}{\sqrt{n}}$ devem ser discutidas. A utilização deste último, o que depende da estimativa da variância, resultante das n observações, é claramente mais seguro quando não se conhece o valor de $\sigma$. Por outro lado, se $\sigma$ é razoavelmente conhecido na região entre zero e $\mathrm{L}_{\mathrm{D}}(\mathrm{de}$ teoria ou observação), e especialmente se for aproximadamente constante, mais intervalos realistas e limites superiores seria definido através da sua utilização, em vez de $\overline{L_{D}}+t_{1-\gamma} \frac{s}{\sqrt{n}}$. Naturalmente, $\frac{\sigma}{\sqrt{n}}$ substitui $\sigma$, quando $\bar{S}$ é utilizado no lugar de S. A estimativa $\frac{\mathrm{s}}{\sqrt{\mathrm{n}}}$ é coerente com $\frac{\sigma}{\sqrt{\mathrm{n}}}$ se o processo de medição permanece sob controle. Assim, é indiferente escolher um em detrimento do outro, principalmente, quando o processo de medição inclui as n observações. Neste caso, o desvio padrão da média das $\mathrm{n}$ observações, seria simplesmente, $\frac{\mathrm{s}}{\sqrt{\mathrm{n}}}$, em vez de $\frac{\sigma}{\sqrt{\mathrm{n}}}[5]$. Apesar da literatura (WINEFORDNER; LONG, 1983; HELSEL, 1990; YU et al., 2002; ALLEGRINI; OLIVIERI, 2014) apresentar diferentes modelos estatísticos que são utilizados na estimativa do limite de detecção, o trabalho de Currie (1968) tornou-se referencial para todos eles. Como pode ser observado na Tabela 1, existe grande variabilidade no conjunto de dados analisados (Figura 12). Isto dificulta a análise estatística em decorrência da presença dos valores anômalos. Apesar da existência de valores outliers (Figura 13), os 
dados tendem para a distribuição de probabilidade normal. A Figura 14 mostra a distribuição dos dados bootstrap para 2000 iterações, enquanto a Figura 15 mostra o ajuste dos dados bootstrap à distribuição de probabilidade normal.

Observa-se que os dados reamostrados tenderam para a distribuição de probabilidade normal, conforme mostra as Figuras 14 e 15, pois segundo Helene e Vanin (2002), o Teorema do Limite Central garante que, quando " $\mathrm{n}$ " for muito grande, os estimadores da média e dos desvios são normalmente distribuídos e convergem para os seus valores verdadeiros. Assim, a aplicação do método bootstrap aos dados apresentados na Tabela 1, diminuíram significativamente a dispersão no conjunto dos dados. Na Tabela 2 encontram-se os valores da média aritmética experimental e bootstrap.

Tabela 2. Médias aritmética experimental e bootstrap das concentrações de ${ }^{210} \mathrm{~Pb}$.

\begin{tabular}{cc}
\hline Estatística & Concentração de $^{210} \mathbf{P b}\left(\mathbf{m B q} \cdot \mathbf{L}^{-1}\right)$ \\
\hline Média aritmética experimental \pm desvio padrão & $33,64 \pm 21,6$ \\
Média aritmética bootstrap \pm desvio padrão (Boot limit) & $33,92 \pm 5,19$ \\
\hline
\end{tabular}

Figura 12 - Histograma dos valores experimentais das concentrações de ${ }^{210} \mathrm{~Pb}$

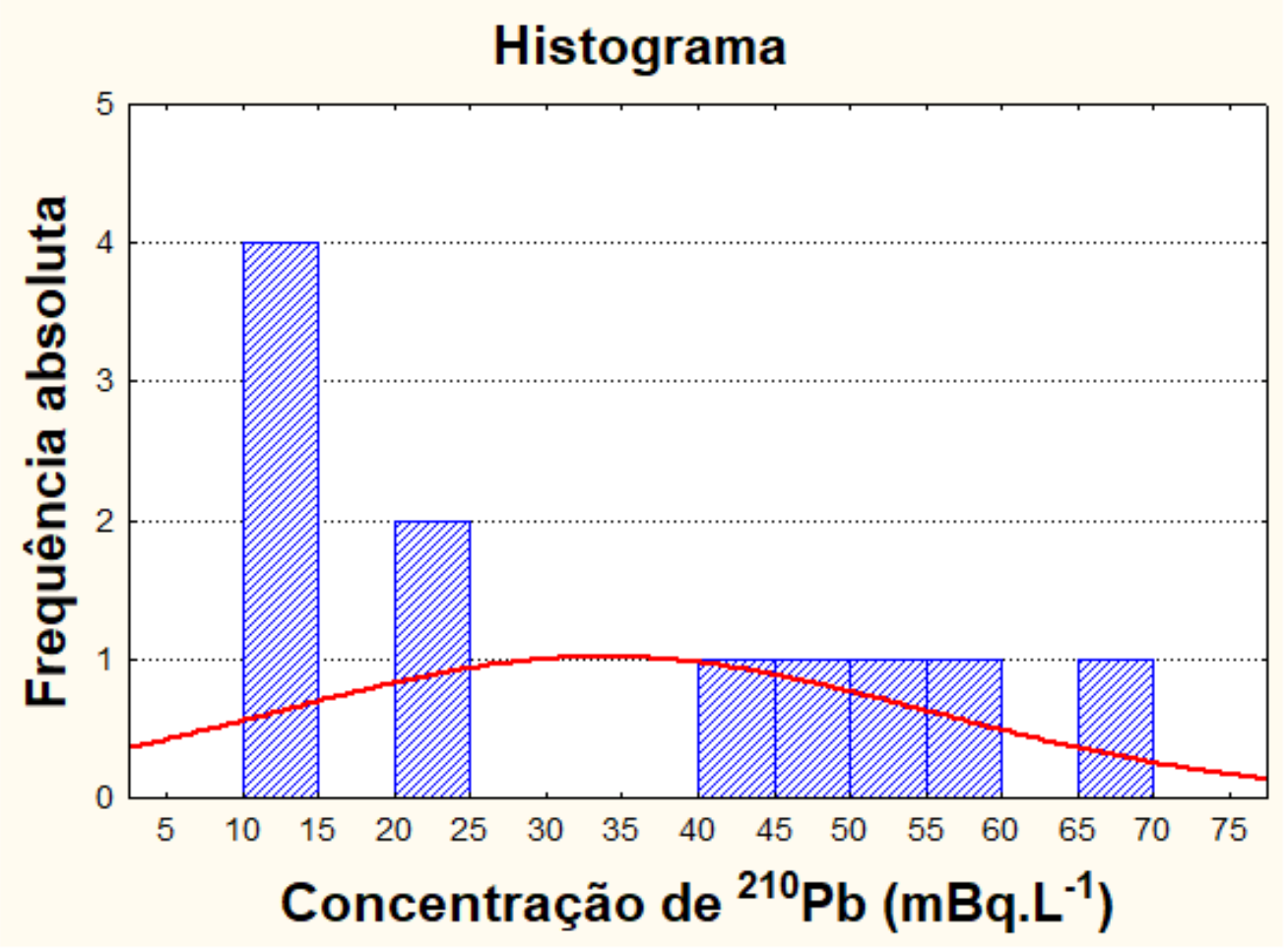


Figura 13 - Ajuste as concentrações experimentais de ${ }^{210} \mathrm{~Pb}$ à distribuição normal

Distribuição de probabilidade normal

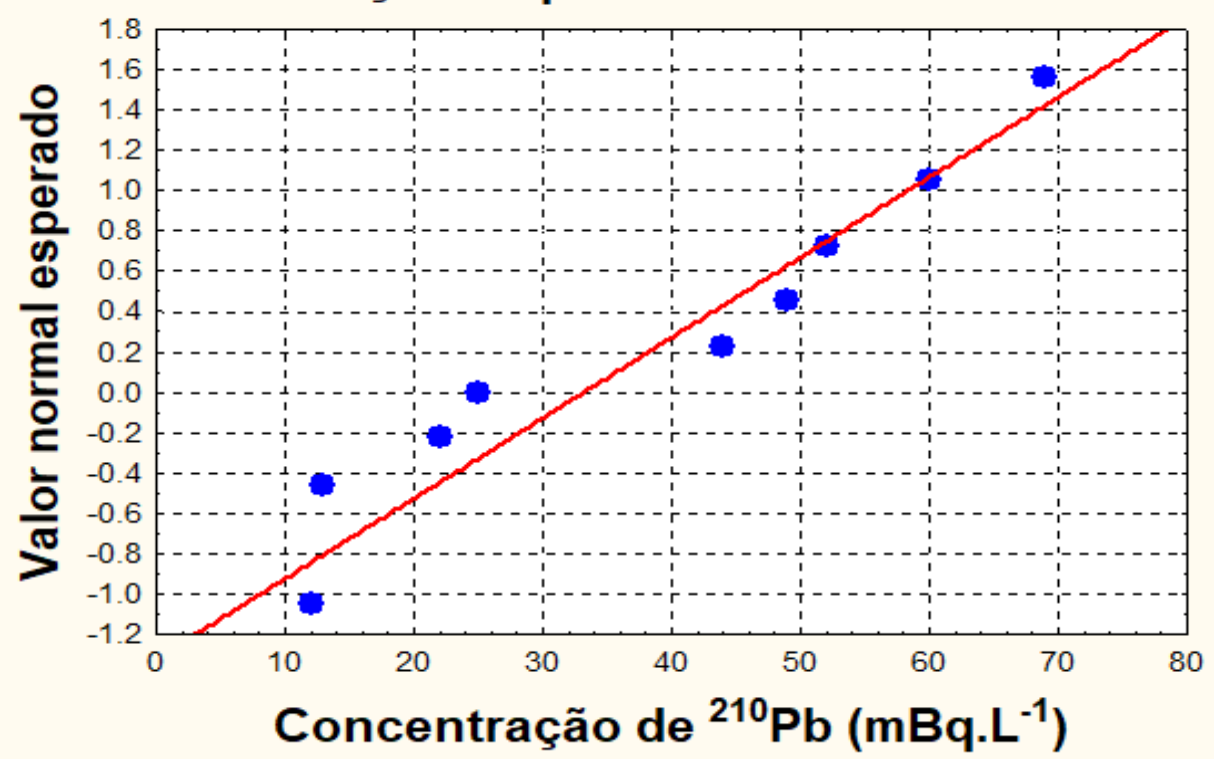

Figura 14 - Histograma dos valores bootstrap das concentrações de ${ }^{210} \mathrm{~Pb}$

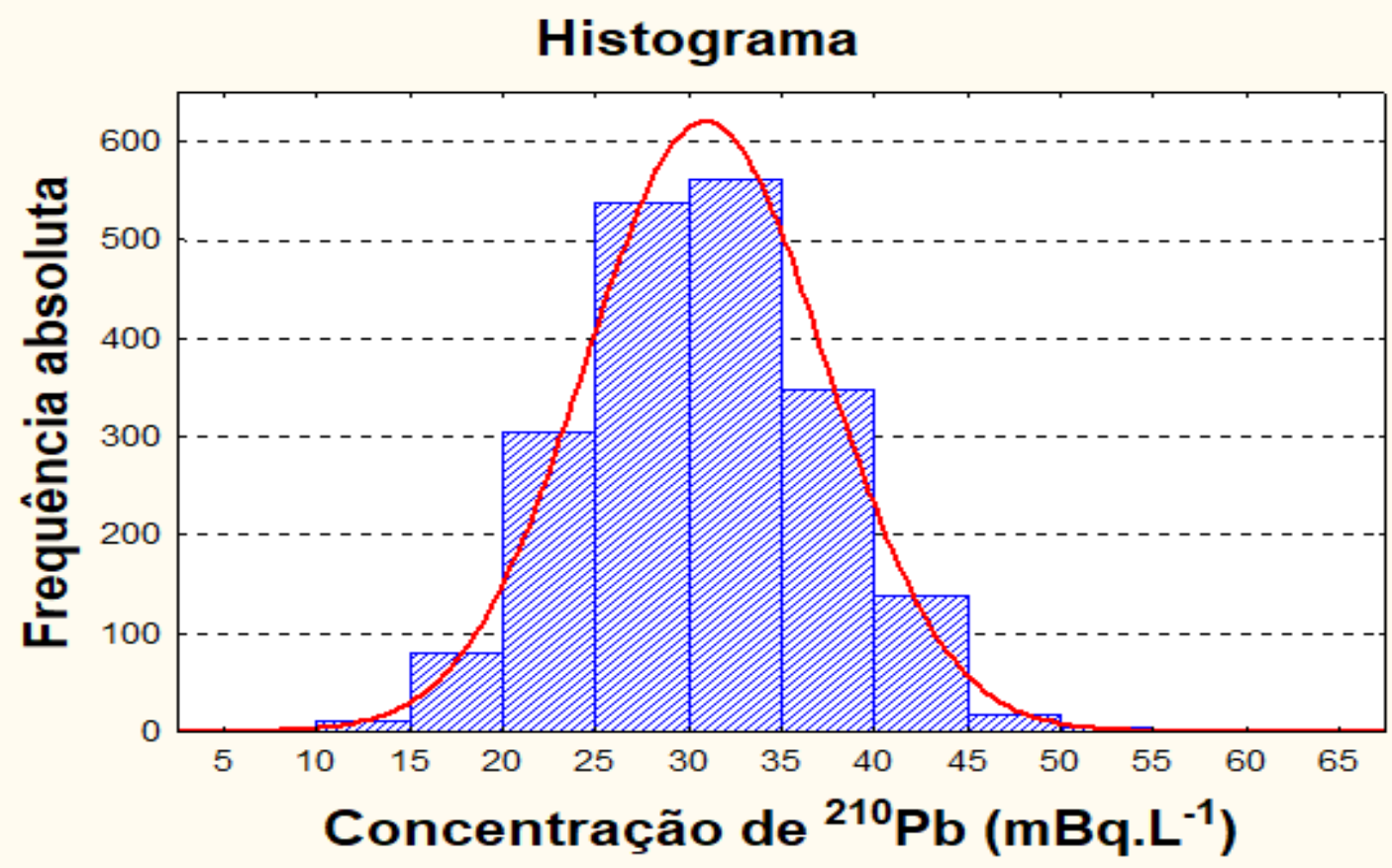


Figura 15 - Ajuste das concentrações bootstrap de ${ }^{210} \mathrm{~Pb}$ à distribuição normal

\section{Distribuição de probabilidade normal}

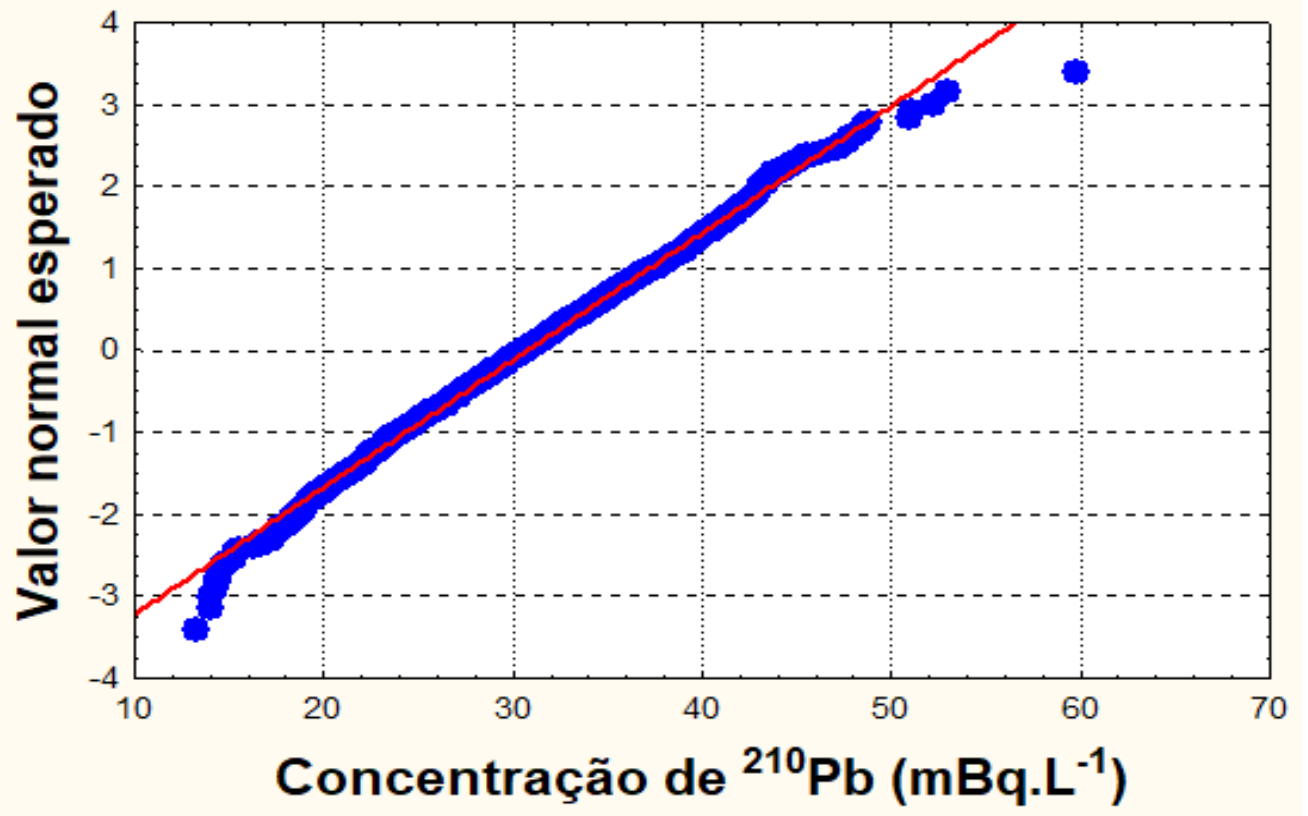

Os resultados apresentados na Tabela 2 mostram elevado grau de dispersão em torno da média aritmética experimental. Assim, a média aritmética experimental não é uma medida de tendência central adequada para representar o conjunto de dados da Tabela 1. A Figura 12 mostra o histograma da distribuição dos valores das concentrações de ${ }^{210} \mathrm{~Pb}$ apresentados na Tabela 1. Observa-se que a distribuição dada pela Figura 12 é bastante achatada, sendo do tipo platicúrtica. Nesse tipo de distribuição, os dados obtidos da amostra encontram-se bastante espalhados, dificultando assim, a escolha adequada de uma medida de tendência central que represente adequadamente o conjunto de dados da Tabela 1. Por outro lado, observa-se que a média aritmética bootstrap é semelhante à média aritmética experimental. Porém, a dispersão em torno da média aritmética bootstrap foi significativamente reduzida (Figura 14). Logo, a média aritmética bootstrap é um estimador não tendencioso da média da população, constituindose assim a medida de tendência central adequada para representar o conjunto de dados da Tabela 1. Portanto, a distribuição bootstrap foi amplamente utilizada em todas as operações estatísticas usadas na construção dos intervalos de confiança para o limite de detecção, considerando os dados contidos na Tabela 1.

Considerando os estudos realizados por Currie (1968), em relação ao valor crítico $\mathrm{L}_{\mathrm{C}}$ como sendo o nível do sinal líquido (resposta do instrumento), acima do qual um sinal observado $\mathrm{S}$ pode ser reconhecido de forma confiável como "detectado", é possível estabelecer conexões entre a definição de $\mathrm{L}_{\mathrm{C}}$ e os dados apresentados na Tabela 1. Sendo assim, pode-se considerar que os valores da Tabela 1 comportam-se como valores críticos. Então, a Figura 14 mostra a distribuição dos valores críticos para o cálculo do limite de detecção. Fazendo os cálculos, considerando as Equações 7 e 8, conforme proposto por Currie (1968), e tomando os dados da Tabela 1 como valores críticos, obtemos como resultados os valores do limite de detecção, como apresentado na Tabela 3.

Apesar dos valores apresentados na Tabela 3 terem sidos gerados usando o desvio padrão da distribuição bootstrap, observa-se ainda grande variabilidade no conjunto de dados do limite de detecção. Isto pode ser observado nas Figuras 16 e 17. Porém, em relação às Figuras 18 e 19, observa-se que, 
quando o método bootstrap foi aplicado aos valores dos limites de detecção da Tabela 3, a variabilidade no conjunto de dados diminuiu significativamente. Isto mostra mais uma vez, a grande vantagem de se utilizar a distribuição de probabilidade normal bootstrap na determinação de limite de detecção, em relação à distribuição normal clássica. Na Tabela 4 encontram-se apresentados os valores das médias aritmética experimental e bootstrap do limite de detecção.

Tabela 3. Valor crítico e limite de detecção.

\begin{tabular}{cc}
\hline Valor crítico $\left(\mathbf{m B q} \cdot \mathbf{L}^{-\mathbf{1}}\right)$ & Limite de detecção $\left(\mathbf{m B q} \cdot \mathbf{L}^{\mathbf{- 1}}\right)$ \\
\hline 12 & 26,7 \\
25 & 52,7 \\
13 & 28,7 \\
12 & 26,7 \\
52 & 106,7 \\
12 & 26,7 \\
44 & 90,7 \\
22 & 46,7 \\
60 & 122,7 \\
49 & 100,7 \\
69 & 140,7 \\
\hline
\end{tabular}

Tabela 4. Médias aritmética experimental e bootstrap do limite de detecção.

\begin{tabular}{cc}
\hline Estatística & Limite de detecção $\left(\mathbf{m B q} \cdot \mathbf{L}^{\mathbf{- 1}}\right)$ \\
\hline Média aritmética experimental \pm desvio padrão & $70,00 \pm 43,2$ \\
Média aritmética bootstrap \pm desvio padrão (Boot limit) & $70,34 \pm 10,0$ \\
\hline
\end{tabular}

Observa-se na Tabela 4, que a média aritmética bootstrap foi praticamente igual à média aritmética experimental. Entretanto, a média aritmética bootstrap mostrou-se bastante resistente às flutuações existentes no conjunto de dados dos valores do limite de detecção apresentado na Tabela 3. Assim, a média aritmética bootstrap foi a medida de tendência central mais adequada para representar o valor do limite de detecção. Os intervalos de confiança para as médias aritmética experimental e bootstrap encontram-se apresentados na Tabela 5.

Tabela 5. Intervalo de confiança para as médias aritmética experimental e bootstrap.

\begin{tabular}{cc}
\hline Estatística & Intervalo de confiança (mBq. $\left.\mathbf{L}^{\mathbf{- 1}}\right)$ \\
\hline Média aritmética experimental & {$[44,4 ; 95,5]^{*}$} \\
Média aritmética bootstrap (Boot limit) & {$[45,3 ; 89,3]$} \\
\hline \multicolumn{2}{c}{ *Com nível de confiança de 95\%. }
\end{tabular}


Figura 16 - Histograma dos valores experimentais do limite de detecção

\section{Distribuição de probabilidade normal}

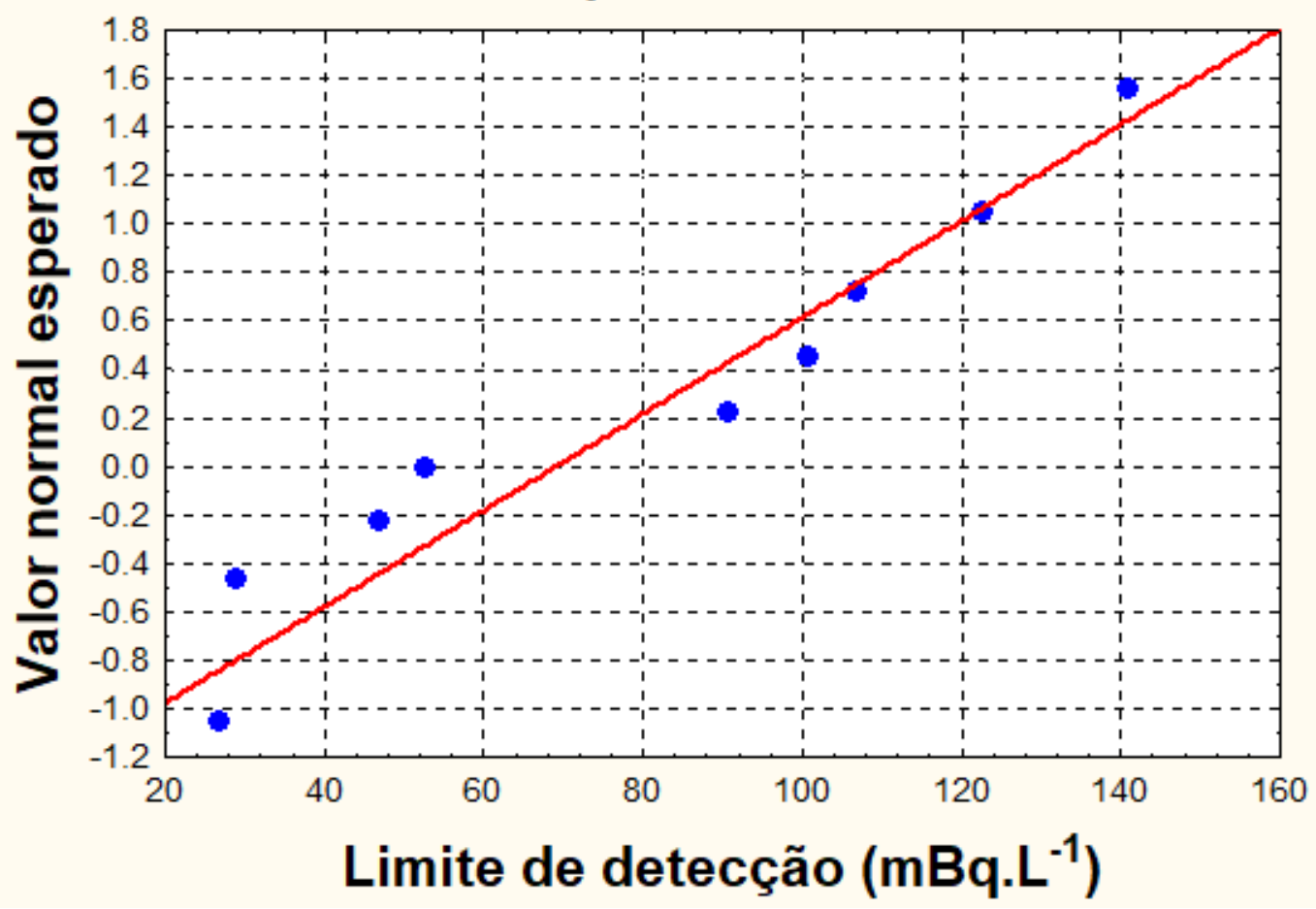

Figura 17 - Ajuste dos valores experimentais do limite de detecção à distribuição normal

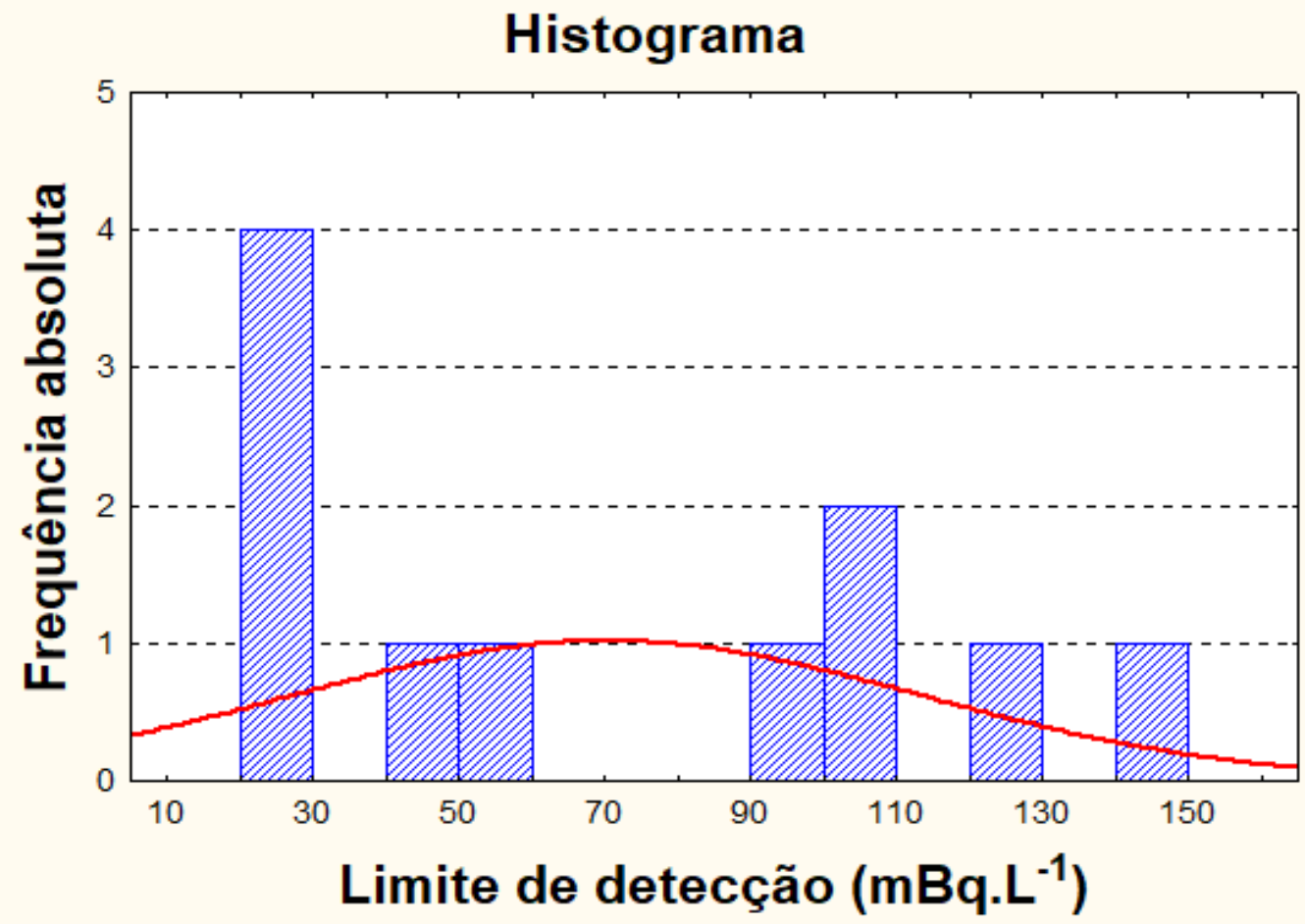


Figura 18 - Histograma dos valores bootstrap do limite de detecção

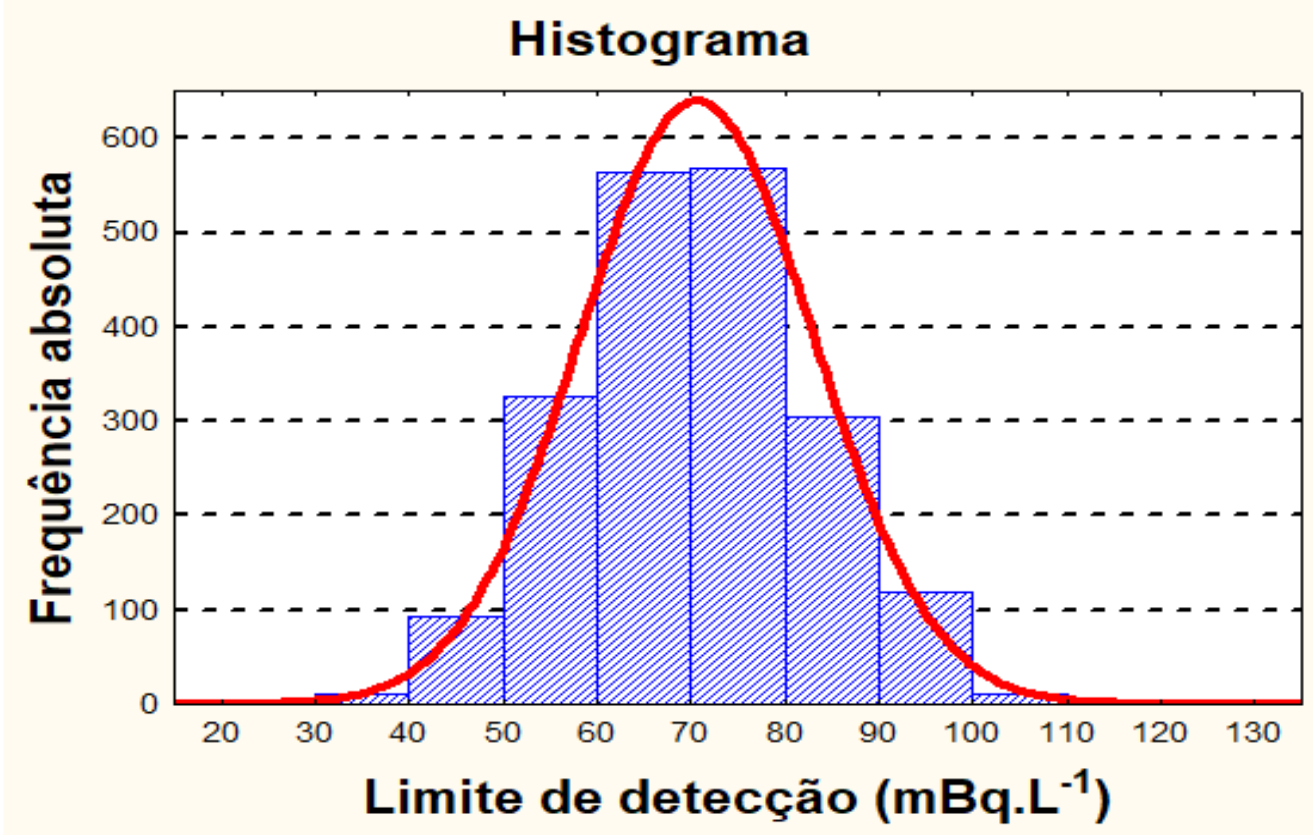

Figura 19 - Ajuste dos valores bootstrap do limite de detecção à distribuição normal

\section{Distribuição de probabilidade normal}

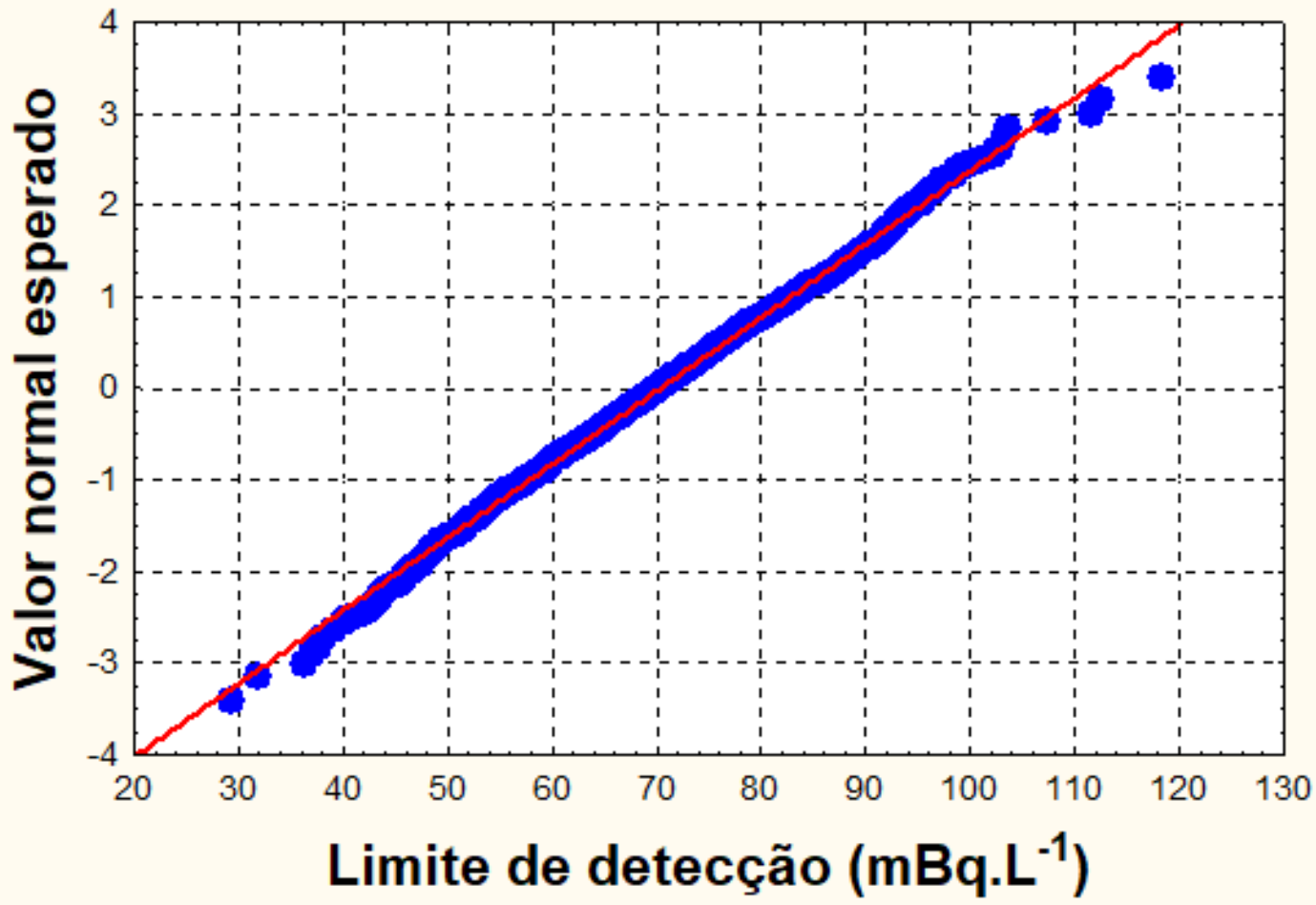

Como pode ser observado na Tabela 5, o intervalo de confiança foi mais amplo para a média aritmética experimental do que para a média aritmética bootstrap. Isto mostra maior grau de dispersão nos valores do limite de detecção da amostra experimental (Figuras 16 e 17). De acordo com Arango (2005), o desvio padrão é um fator importante que influência a amplitude do intervalo de confiança. Assim, quanto maior for o desvio padrão (Figuras 16 e 17), maior será a amplitude do intervalo. A tomada de decisão 
sobre o limite de detecção em pesquisas radioquímicas deve ser realizada de tal maneira que o intervalo de confiança a ser determinado seja proveniente de rígidos processos de controle de qualidade experimental e estatístico. Somente assim, será possível evitar que valores de concentrações de um dado analito que se encontram próximos do limite de detecção, não sejam incorporados ao conjunto de dados experimentais.

\section{CONCLUSÃO}

Os resultados mostraram que o método bootstrap é uma ferramenta estatística bastante eficiente para construir intervalos de confiança para limite de detecção em estudos radioquímicos. A distribuição de probabilidade bootstrap mostrou-se mais robusta do que a distribuição de probabilidade normal clássica na análise estatística inferencial, quando utilizada na determinação do limite de detecção. As medidas de tendência central bootstrap são excelentes estimadores para a média aritmética dos valores dos limites de detecção. O programa boot limit pode ser utilizado em estudos radioquímicos na análise estatística inferencial para diferentes tipos de dados, inclusive em conjuntos de dados contendo valores outliers.

\section{REFERÊNCIAS}

AGÊNCIA NACIONAL DE VIGILÂNCIA SANITÁRIA (ANVISA). Resolução 899 de 29 de maio de 2003 - Guia para Validação de Métodos Analíticos e Bioanalíticos.

ALLEGRINI, F.; OLIVIERI, A.C. IUPAC-Consistent Approach to the Limit of Detection in Partial LeastSquares Calibration. Anal Chem, v. 86, p. 7858-7866, 2014.

ALTSHULER, B.; PASTERNACK, B. Statistical Measures of the Lower Limit of Detection of a Radioactivity Counter. Health Physics, v. 9, p. 293-298, 1963.

ARANGO, H. G. Bioestatística: teórica e computacional. Guanabara Koogan, Rio de Janeiro, 2 ed., 2005.

BICKEL, P.J.; FREEDMAN, D.A. Some asymptotic theory for the bootstrap. Annals of Applied Statistics, v. 9, p. 1196-1217,1981.

CURRIE, L.A. 1968. Limits for Qualitative Detection and Quantitative Determination: Application to Radiochemistry. Analytical Chemistry, v. 40, n. 3, p.586-593, 1968.

EFRON, B. The jackknife, the bootstrap and other resampling plans. Bristol: J.W. Arrowsmith, Ltd, 1982.

EFRON, B., TIBSHIRANI, R.J. An introduction to the bootstrap. New York: Chapman e Hall, 1993.

GODOY, J.M. et al.An alternative method for the determination of excess ${ }^{210} \mathrm{~Pb}$ in sediments. Radiation Protection Dosimetry, v. 75, p.111-115, 1998.

HELENE, O., VANIN, V. R. Analysis of discrepant data using a bootstrap procedure. Nuclear Instruments and Methods in Physics Research Section A: Accelerators, Spectrometers, Detectors and Associated Equipment, v. 481, p. 626-631, 2002.

HELSEL, D.R. Less than obvious: Statistical treatment of data below the detection limit. Environ. Science Technology, v. 24, n. 12, p.52-59, 1990. 
INMETRO DOQ-CGCRE-008 - março de 2003 - Orientações sobre Validação de Métodos de Ensaios Químicos, Revisão 01, 35p., 2003.

INTERNATIONAL ORGANIZATION FOR STANDARDIZATION (ISO). Determination of the characteristic limits (decision threshold, detection limit and limits of the confidence interval) for measurements of ionizing radiation. Fundamentals and application. BS ISO 11929:2010 (Geneva:ISO).

INTERNATIONAL UNION OF PURE AND APPLIED CHEMISTRY (IUPAC). Nomenclature in Evaluation of Analytical Methods Including Detection and Quantification Capabilities. Pure and Applied Chemistry, v. 67, n. 10, p.1699-1723, 1995.

JIA, G., TORRI, G. Determination of ${ }^{210} \mathrm{~Pb}$ and ${ }^{210} \mathrm{Po}$ in soil or rock samples containing refractory matrices. Applied Radiation and Isotopes, v. 65, p.1-8, 2007.

MONTGOMERY, D.C. Introduction to statistical quality control. 6th edition. Arizona: John Wiley \& Sons, Inc., 2009.

MURTEIRA, B. J. F. Probabilidades e estatística. Lisboa: McGraw-Hill, 2 ed, 1990.

NICHOLSON, W.L. Fixed Time Estimation of Counting Rates with Background Corrections. AEC Research and Development Report HW-76279, 1963.

NICHOLSON, W.L. Statistics of Net-counting-rate Estimation with Dominant Background Corrections. Nucleonics, v. 24, n. 8, p.118-121, 1966.

SILVA, A.N.C., AMARAL, R.S., SANTOS JÚNIOR, J.A. WILSON VIEIRA, J.W., MENEZES, R.S.C. Statistical analysis of discrepant radioecological data using Monte Carlo Bootstrap Method. Journal of Radioanalytical and Nuclear Chemistry, v. 306, n.3, p. 571-577, 2015.

SILVA, C.M.et al. Application of bootstrap method for evaluating discrepant levels of radium-226 in forage palm (Opuntia spp). Revista Brasileira de Biometria, v.25, n.3, p.109-114, 2007.

SILVA, C.M. et al. Pb-210 in Rock and Soils of the Semi-Arid Agreste Region of Pernambuco, Brazil. Bulletin of Environmental Contamination and Toxicology, v. 82, p. 647-649, 2009.

VIEIRA, J.W. Construção de um modelo computacional de exposição para cálculos utilizando o código monte carlo egs4 e fantomas de voxels. Tese de Doutorado do Programa de Pós-graduação em Tecnologias Energéticas e Nucleares da Universidade Federal de Pernambuco, 2004. 146p.

WEHRY, E. Quantitative Measurements. In: SETTLE, F. (Ed.). Handbookof Instrumental Techniques of Analytical Chemistry. Upper Saddle River: Prentice Hall, 1997.

WINEFORDNER, J.D; LONG, G.L. Limit of detection: A closer look: at the IUPAC definition. Analytical Chemistry, v. 55, n. 7, 1983.

YU, L.L., FASSETT, J.D., GUTHRIE, W.F. Detection Limit of Isotope Dilution Mass Spectrometry. Analytical Chemistry, v. 74, p. 3887-3891, 2002. 